\title{
Operational space control of a lightweight robotic arm actuated by Shape Memory Alloy wires: a comparative study
}

\author{
Serket Quintanar-Guzmán, Somasundar Kannan, Adriana Aguilera-González, Miguel A. \\ Olivares-Mendez and Holger Voos
}

\begin{abstract}
This paper presents the design and control of a two-link lightweight robotic arm using Shape Memory Alloy wires as actuators. Both, a single wire actuated system and an antagonistic configuration system are tested in open and closed-loop. The mathematical model of the SMA wire, as well as the kinematics and dynamics of the robotic arm, are presented. The Operational Space Control of the robotic arm is performed by using a Joint Space control in the inner loop and Closed Loop Inverse Kinematics in the outer loop. In order to choose the best Joint Space Control approach, a comparative study of four different control approaches (Proportional Derivative, Sliding Mode, Adaptive and Adaptive Sliding Mode Control) is carried out for the proposed model. From this comparative analysis, the adaptive controller was chosen to perform Operational Space Control. This control helps us to perform accurate positioning of the endeffector of SMA wire based robotic arm. The complete Operational Space control was successfully tested through simulation studies performing position reference tracking in the end-effector space. Through simulation studies the proposed control solution is successfully verified to control the hysteretic robotic arm.
\end{abstract}

\section{Keywords}

SMA wire, Adaptive Control, Lightweight Robotic Arm, Operational Space position control, hysteresis.

\section{Introduction}

Shape Memory Alloys (SMA) are a type of smart materials that can "remember" their original shapes. These type of alloys have the ability to recover its original pre-defined shape by applying certain stimuli such as thermo-mechanical variations. This phenomenon is known as Shape Memory Effect (SME)(Rao et al., 2015). The SME occurs due to an inner transformation of the material's crystalline structure. This transformation happens between two phases called martensite and austenite. When the SMA wire is at lower temperature its structure shifts to the martensite phase which is a relatively soft and malleable phase, during which the wire can be easily deformed. When heated over the transformation temperature, the SMA wire transforms back into the austenite phase, a hard phase, recovering its initial form and size (Rao et al., 2015).

Among the more common SMAs one can find for example Nickle-Titanium, Gold-Cadmium and Copper-Zinc-Aluminium. Where the most used one has been the Nickel-Titanium alloy (which is also known as Nitinol) (Zheng et al., 2014). There are several physical properties of Nitinol being studied and tested, such as: shape memory, pseudo-elasticity, corrosion resistance, magnetic susceptibility, damping, mass ratio, small size, noiseless operation, heat capacity, bio-compatibility, thermal conductivity, and other mechanical properties including hardness, impact toughness, fatigue strength and machinability. These properties make SMA wires ideal for applications such as biomedical and dental implants, aerospace, engineering and sports equipment, among others.

SMAs have drawn significant attention and interest since a few decades. However, it was not until

Interdisciplinary Centre for Security, Reliability and Trust (SnT), University of Luxembourg, LU

\section{Corresponding author:}

Serket Quintanar-Guzmán, SnT, University of Luxembourg, 6 rue Richard Coudenhove-Kalergi , L-1359, Luxembourg, LU.

Email: serket.quintanar@uni.lu 
recent years when the term shape memory technology (SMT) was introduced and a wide range of SMA wires' applications started to be developed. An example of these applications is the bioinspired micro-robots manufacturing, where SMAs are considered as a good alternative to traditional actuators, due to characteristics as corrosion resistance, simple mechanical structure and biocompatibility (Khodayari et al., 2011; Colorado et al., 2011; Gao et al., 2014; Shin et al., 2015). SMA wires have also been used in medical devices like intra-arterial supports (Nematzadeh and Sadrnezhaad, 2012) or wires for suturing (Nespoli et al., 2015), in orthopedic devices as a spinal cage implant (Andani et al., 2015), adaptive anklefoot orthoses (Mataee et al., 2015) or skeletal fixation devices (mandibular segmental) (Moghaddam et al., 2016), as well as dental and orthodontic applications (Jafari et al., 2008; Pandis and Bourauel, 2010). In parallel, SMA wires have also proved to be a good alternative when dealing with aerodynamic problems requiring high-precision coordination, and some solutions have been applied for small prototypes and unmanned aerial vehicles (UAVs). For example in Rodrigue et al. (2016), a morphing segment actuated by multiple embedded SMA wires was implemented in a UAV wing, where the capability to maintain a smooth twisting concentrated on a segment of the wing was tested with a prototype. Similarly, it is possible to include as well the work of Barbarino et al. (2009), which presents a wing shape control using SMA wires as actuation devices to produce a local bump. Furthermore, Kennedy et al. (2004) presented a blade actuator that is developed for the helicopter blade-tracking problem, which utilizes the SMA as the active actuator material to drive a rotor blade trim tab for the purpose of maintaining rotor tracking. All these articles and several others propose solutions to improve the aerodynamic properties of the flying devices.

Among the many applications of the SMA wires, several specific purpose actuators have been reported in the literature, such as: construction vibrations dampers (Sreekumar et al., 2009), camera lens focus actuators (Son et al., 2009), car mirror actuators (Williams et al., 2010) or SMA based motors (Quintanar-Guzmán et al., 2014). Moreover, SMA wires are also useful in robotic manipulators since they allow motion without using larger drives. For instance, the human-like robotic arm developed by Hulea and Caruntu (2014), where a neural network control for artificial muscles was implemented on a robotic arm joint using a SMA wire as actuator. Another example is given by Ko et al. (2011), where the authors propose a fuzzy-PID control of an anthropomorphic artificial finger actuated by three antagonistic SMA muscle pairs. In addition to the aforementioned, multiple general purpose actuators have been developed for micro-positioning applications using advanced control techniques (Kannan et al., 2010, 2013; Kannan, 2011).

Nonetheless, most of the mentioned applications are micro-scale or require complicated mechanical systems to be implemented. For this reason, in a previous publication the design of a SMA wire actuated robotic arm was presented (Quintanar-Guzmán et al., 2016). This proposal seeks to keep the simplicity of the mechanics and therefore achieves a lightweight actuator capable of producing a relevant amount of force levels, leading to a suitable performance per weight ratio. This lightweight characteristic is critical in applications like robotic manipulators for UAVs, where the optimal use of available payload is a great challenge. This implementation will be the main purpose of this arm. With this in mind, we propose a suitable controller for the lightweight robotic arm design, which enables the arm to be implemented without significantly decreasing the quadcopter's available payload.

This paper contains two main contributions: first, a comparative analysis between two different configurations of the robotic arm is presented, in order to study the nonlinearities of SMA wires as hysteresis, saturation and dead zone. Second, the development of a control concept which is capable of dealing with the nonlinear dynamics of SMA wires is discussed. Along with slow dynamics, the nonlinear response of the SMA wire entails a huge challenge for implementations that require high accuracy or fast response. For this purpose, a comparative analysis among four different control approaches is presented: Proportional Derivative (PD) control, Sliding Mode Control (SMC), Adaptive Control and Adaptive Sliding Mode Control (ASMC) is presented. These controllers are compared with the aim to find a suitable controller for the proposed lightweight robotic arm. The selected controller is then used for the development of an operational space control for position regulation of the end-effector.

The remaining of this paper is organized as follows: First we present the mechanical design and mathematical model of the proposed lightweight robotic arm, followed by a comparison between the two different joint configurations proposed for the actuator (biased SMA wire and two antagonistic wires). Subsequently four different control approaches in joint space are developed and analysed, continued by 
a thermal disturbance analysis. After, an operational space control law for position regulation of the endeffector is applied. Finally we conclude with the discussion of the results and possible future works.

\section{Basic structure of the SMA actuated robotic arm}

In this section we present the mechanical design of a lightweight SMA actuated robot arm in two different possible configurations: single biased wire and antagonistic wires.

The optimal use of available payload of an aerial vehicle is critical for the design of aerial manipulators. With this in mind, we propose a single Degree of Freedom (DOF) actuator wich is actuated by SMA wires. In the Figure 1, a Computer Aided Design (CAD) model of the robot arm design is shown. The design is based on an existing joint proposed in Guo et al. (2015), which consists of two couplers joined by a torsion spring. This design allows to select between a single wire actuated system or an antagonistic configuration.

In the first configuration (biased single wire) the SMA wire is attached at one end to the coupler-1, while the second coupler is fixed with a hard wire, i.e., only one wire is a SMA wire. With this design, the actuator behaves like a biased SMA wire. Here, the SMA-1 affects directly the angular position of the end effector $\left(\theta_{1}\right)$ by controlling the angular position of coupler- 1 (see Figure 1) while the deformation force is applied by the torsion spring and is directly proportional to the position of coupler-1.

For the second configuration (antagonistic wires) each wire is attached at one end to its respective coupler, allowing to control independently the angular position of each coupler. Controlling the second coupler's position allows to adjust the torque applied by the torsion spring and hence, increase or decrease the overall stiffness of the joint as required. This change in stiffness entails a change in the transformation temperatures, so the wire's transformation could be hastened in a controlled manner. The following sections present a more detailed analysis of the characteristics of these two designs.

The given robotic arm with 1 DOF, is activated by two $37 \mathrm{~cm}$ long Nitinol wires. It has two custom-made carbon fiber links (150 $\mathrm{mm}$ and $100 \mathrm{~mm}$ respectively) and the range of movement along the vertical plane $X-Z$ is up to 85 degrees with two $7.5 \mathrm{~mm}$ radius couplers. It has a total weight of $48 \mathrm{~g}$, which is only about $25 \%$ of the weight of other lightweight designs found in the literature, such as the one presented

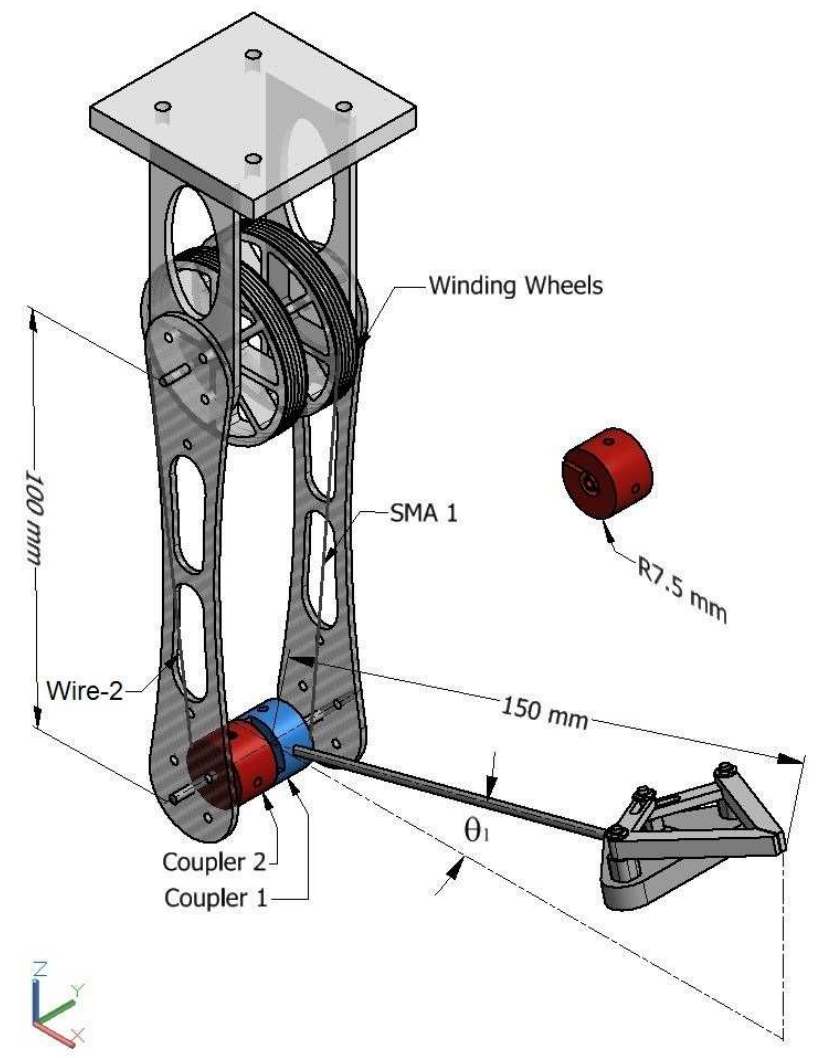

Figure 1. Proposed SMA wire actuated robotic arm CAD model.

in Bellicoso et al. (2015). The winding wheels enable the use of longer SMA wires in order to increase the movement range without increasing the dimension of the links. It is important to emphasize that an increase in the length of the wires will increase the energy consumption. For this reason a balance between range of motion and energy consumption should be considered, especially when considering a mobile application like aerial manipulation.

\section{Model of the SMA actuated robotic arm}

In this section the mathematical model of the overall system is discussed. The mathematical model of the SMA wires, as well as the kinematics and dynamics of the arm resulting from the mechanical design are explained in detail.

Figure 2 shows the block diagram of the robotic arm's mathematical model for both configurations: a) shows the single wire biased configuration, and b) the antagonistic configuration. The robotic arm model consist of two main subsystems: 1) the SMA wire model and 2) the Kinematic and Dynamic model. 

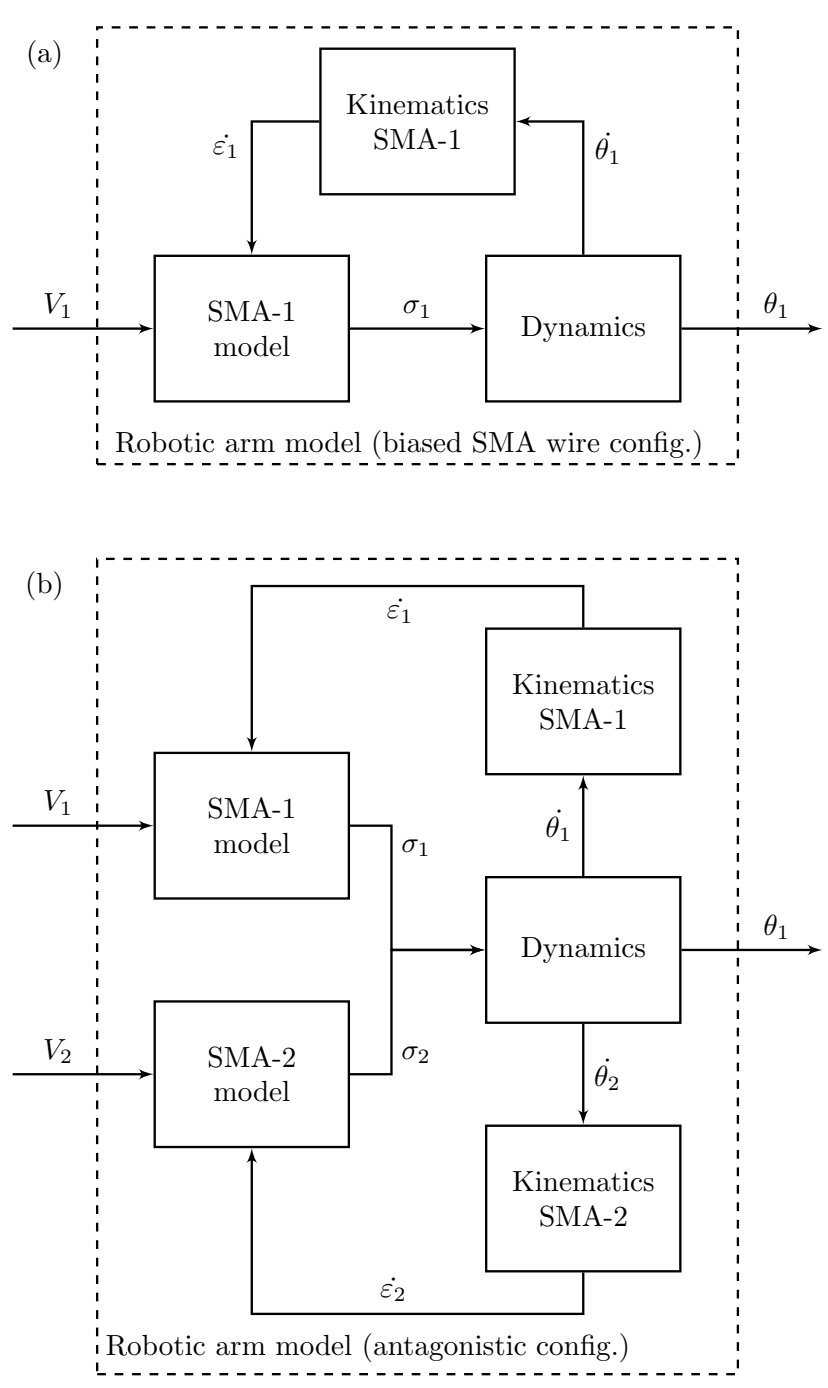

Figure 2. Block diagram of the SMA actuated robotic arm, (a) biased wire configuration; (b) antagonistic configuration.

\section{SMA wire subsystem}

The schematic model describing the SMA wire subsystem is illustrated in the Figure 3. This subsystem is described by a mathematical model of the Nitinol wire which was proposed by the authors in Elahinia and Ashrafiuon (2002). This is likewise divided into three subsystems representing the thermal dynamics, the heat transformation and the constitutive model. In the Figure 3 the interaction between the variables of each subsystem of the SMA wire model is shown. On the other hand, the dynamics of the arm are directly derived from a CAD model. Each of the mentioned subsystems will be explained in more detail in the following subsections.

Heat transfer model. This block consists of the electrical heating (Joule effect) and the natural

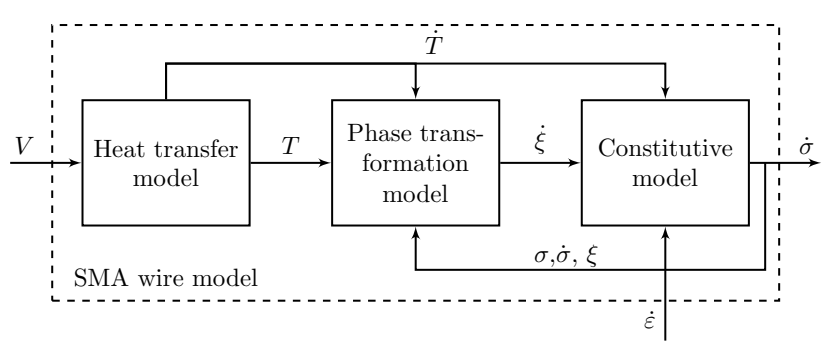

Figure 3. SMA wire mathematical model block diagram.

convection model described by the following equation (Elahinia and Ashrafiuon, 2002):

$$
m_{w} c_{p} \frac{d T}{d t}=\frac{V^{2}}{R}-h A_{w}\left(T-T_{a m b}\right)
$$

where $V$ is the voltage, $R$ is the electric resistance per unit length, $c_{p}$ is the specific heat, $m_{w}$ is the mass per unit length, $A_{w}$ is the wire surface area, $T_{a m b}$ the ambient temperature and $T$ is the SMA wire temperature. Here $h$ is approximated by a second order polynomial of the temperature:

$$
h=h_{0}+h_{2} T^{2}
$$

SMA wire phase transformation model. As shown in the Figure 3, the block containing the phase transformation model (from martensite to austenite) computes the martensite fraction $(\xi)$. The phase transformation of the SMA wire depends directly on the direction of the time derivative of the temperature. Therefore, due to hysteresis behavior two equations are needed to fully describe this phenomenon. This phase transformation while heating is given by (Elahinia and Ashrafiuon, 2002):

$$
\xi=\frac{\xi_{M}}{2}\left\{\cos \left[a_{A}\left(T-A_{s}\right)+b_{A} \sigma\right]+1\right\}
$$

for $A_{s}+\frac{\sigma}{C_{A}} \leq T \leq A_{f}+\frac{\sigma}{C_{A}}$.

Inversely, the transformation from austenite to martensite, during cooling is described by the following equation (Elahinia and Ashrafiuon, 2002):

$$
\xi=\frac{1-\xi_{A}}{2} \cos \left[a_{M}\left(T-M_{F}\right)+b_{M} \sigma\right]+\frac{1+\xi_{A}}{2}
$$

for $M_{s}+\frac{\sigma}{C_{M}} \leq T \leq M_{f}+\frac{\sigma}{C_{M}}$, where $M_{s}, M_{f}, A_{s}, A_{f}$ are the start and end transformation temperatures for martensite and austenite transformation respectively. Here $a_{A}=\frac{\pi}{\left(A_{f}-A_{s}\right)}, a_{M}=\frac{\pi}{\left(M_{s}-M_{f}\right)}, b_{A}=-\frac{a_{A}}{C_{A}}$, $b_{M}=-\frac{a_{M}}{C_{M}}, C_{A}$ and $C_{M}$ are curve fitting parameters. 
Wire constitutive model. This model describes the relation between stress $\sigma$, strain $\varepsilon$, temperature $T$ and martensite fraction $\xi$. The general form, firstly proposed by Liang and Rogers (1990) and then modified by Elahinia and Ashrafiuon (2002), is written as

$$
\dot{\sigma}=E \dot{\varepsilon}+\Omega \dot{\xi}+\Theta \dot{T},
$$

where $\Omega$ and $\Theta$ represent the phase transformation constant and thermal expansion coefficient, respectively. Herein

$$
\Omega=-E \varepsilon_{0}
$$

and $\varepsilon_{0}$ is the initial strain. The authors of Elahinia and Ashrafiuon (2002) propose a constant value for the Young's modulus $E$ as an average of the Young's modulus of each phase, austenite $\left(E_{A}\right)$ and martensite $\left(E_{M}\right)$. However, since one of the configurations of the actuator presented here uses the antagonistic SMA wires configuration, the Young's modulus cannot be constant since it depends on the stress applied over each wire, which in turn depends on the martensite fraction as follows (Guo et al., 2015):

$$
E=\xi E_{M}+(1-\xi) E_{A}
$$

\section{Kinematic and dynamic model}

In this section the model of the mechanical design (corresponding to the kinematic and dynamic model) and its relation with the rest of the system is explained.

Kinematic model. This model relates the SMA wire model with the mechanics of the robotic arm itself. The strain ratio of the SMA wire and angular velocity of the arm depends on the geometry of the design. This kinematic relation is given as:

$$
\dot{\varepsilon}=-\frac{r \dot{\theta}}{l_{0}}
$$

where $r$ is the coupler radius, $l_{0}$ the initial length of each wire and $\dot{\theta}$ the angular velocity of the coupler. Equation (8) shows that the angular position of each coupler with respect to the $X$-axis $(\theta)$ is inversely proportional to the strain of the wire $(\varepsilon)$.

Dynamic model. The dynamic model used here describes the relation between coupler mechanism, torsion spring and forces applied by the SMA wires, as well as the effects of the load and grip at the end of the second link. The general dynamic model of the mechanical system is described as:

$$
M(\theta) \ddot{\theta}+V_{m}(\theta, \dot{\theta}) \dot{\theta}+g(\theta)+F_{d} \dot{\theta}+\Phi\left(\theta, \theta_{r}\right)=\tau_{\omega}
$$

where $\theta, \dot{\theta}, \ddot{\theta}$ represent the positions, velocities and accelerations of the couplers, $M(\theta)$ is the inertia matrix, $V_{m}(\theta, \dot{\theta})$ is the centripetal-coriolis matrix, $g(\theta)$ is considered as the effect of gravity, $F_{d}$ is the viscous coefficient term, $\Phi\left(\theta, \theta_{r}\right)$ is the nonlinear hysteretic term, $\tau_{\omega}$ is the input torque applied to the manipulator joint by the SMA wire.

The dynamic behavior of the couplers, gripper, load and links was directly obtained from the CAD design shown in Figure 1 (this CAD design was developed in the Autodesk/Inventor software tool). This model does not only include the exact geometry of each piece but also masses, inertias and centers of mass necessary for the dynamic analysis. The CAD model is imported via the SimMechanics toolbox in order to obtain a continuous dynamic MATLAB/Simulink model of the mechanical system. On the other hand, the torsion springs and SMA wires torques were obtained from basic physical laws, where the SMA wire's force $\left(F_{w}\right)$ is deduced by inversely proportional realtion to the stress $(\sigma)$, which can be computed by integration of equation $(5)$ :

$$
\tau_{w i}=F_{w i} r_{i}=A \sigma_{i} r_{i}
$$

where $i=1,2$ for SMA-1 and SMA-2, $r$ is the coupler radius and $A$ is the cross-sectional area of the wire. The torsion spring torque $\tau_{s}$ for the single wire configuration is calculated as:

$$
\tau_{s}=k_{s} \theta_{1}+b_{s} \dot{\theta}_{1}
$$

where $k_{s}$ is the spring constant and $b_{s}$ is the spring's friction factor, $\theta_{1}$ is the angular position of coupler- 1 with respect to $X$-axis. While for the second configuration (antagonistic wires) $\tau_{s}$ is given by:

$$
\tau_{s}=k_{s}\left(\theta_{1}-\theta_{2}\right)+b_{s}\left(\dot{\theta}_{1}-\dot{\theta}_{2}\right)
$$

where $\theta_{2}$ is the angular position of the coupler- 2 with respect to $X$-axis.

\section{Comparison between biased wire and antagonistic wires configuration}

A comparison between the performance of the single wire and the antagonistic (two SMA wires) actuated version of the presented system was carried out. This analysis will be discussed in the current section. For comparative reasons open and closed loop tests were conducted.

As mentioned in previous sections, the use of each configuration entails different characteristics for the actuator dynamics. Figure 4 shows the equivalent mechanical model for both configurations. Here we can 


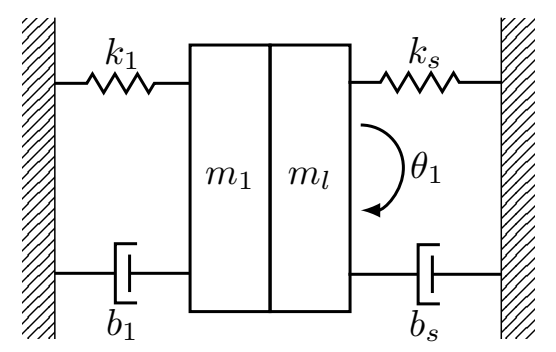

(a) Biased wire

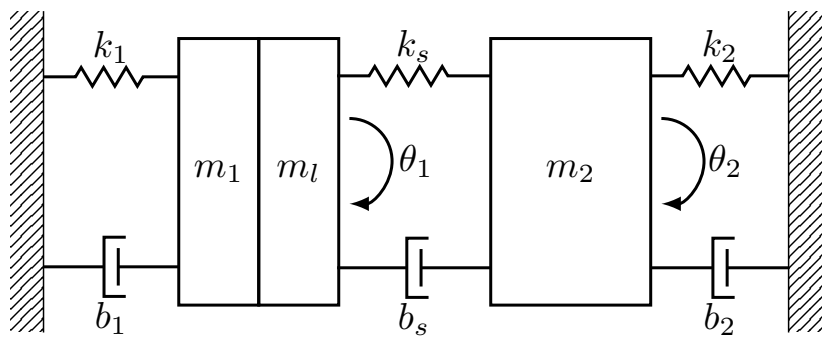

(b) Antagonistic wires

Figure 4. Equivalent mechanical model for SMA actuators: (a) biased SMA wire; (b) antagonistic SMA wires.

observe that for the biased configuration, the torque applied by the torsion spring depends only on the position of the coupler-1 $\left(m_{1}\right.$ and $\left.m_{l}\right)$. On the other hand, when the antagonistic configuration is analyzed, it is clear that the torque of the spring depends on both, coupler-1 and coupler-2 positions. This characteristic provides us with an extra control input that allows accelerating the cooling dynamics of the system.

However, the use of an antagonistic design (2 SMA wires) also increases the complexity for controlling the system as well as the energy consumption. Despite this, as our study will demonstrate, the improvement in velocity of response and accuracy justifies this design decision.

In the Figure 1 the mechanical design of the robotic arm is shown. For the one wire configuration the "Wire-2" represents a hard wire, so the stiffness of the joint cannot be controlled. On the other hand, for the antagonistic configuration a SMA wire is used as "Wire-2", as explained in previous sections.

\section{Open-loop analysis}

The open-loop test is designed to analyze the hysteretic behavior of the system and will allow us to compare the changes in the major hysteresis loop when using the antagonistic configuration. This test was conducted by applying a sinusoidal voltage signal, with the purpose to obtain the main hysteresis loop of Voltage to Strain. In the two SMA wires design, a sinusoidal voltage $180^{\circ}$ shift was applied to the second wire. The value of the parameters of the system model used for simulation are listed in Table 1 and were taken from SMA wire manufacturer in DYNALLOY Inc (2014a,b), Guo et al. (2015) and Elahinia and Ashrafiuon (2002). The gains and parameters corresponding to each controller will be given in the respective subsections. In Figure 5 it is possible to observe the hysteresis loop

Table 1. Parameters of the SMA wire and the compliant actuator.

\begin{tabular}{llll}
\hline Par. & Value & Par. & Value \\
\hline$E_{M}$ & $28 \mathrm{GPa}$ & $C_{A}$ & $10 \mathrm{MPa} /{ }^{\circ} \mathrm{K}$ \\
$E_{A}$ & $75 \mathrm{GPa}$ & $C_{M}$ & $10 \mathrm{MPa} /{ }^{\circ} \mathrm{K}$ \\
$A_{s}$ & $88{ }^{\circ} \mathrm{C}$ & $T_{a m b}$ & $25{ }^{\circ} \mathrm{C}$ \\
$A_{f}$ & $98{ }^{\circ} \mathrm{C}$ & $A$ & $4.9 x 10^{-8} \mathrm{~m}^{2}$ \\
$M_{s}$ & $72{ }^{\circ} \mathrm{C}$ & $A_{w}$ & $290.45 x 10^{-6} \mathrm{~m}^{2}$ \\
$M_{f}$ & $62{ }^{\circ} \mathrm{C}$ & $c_{p}$ & $320 \mathrm{~J} / \mathrm{kg}^{\circ} \mathrm{C}$ \\
$m_{w}$ & $6.8 x 10^{-4} \mathrm{~kg} / \mathrm{m}$ & $\varepsilon_{L}$ & $2.3 \%$ \\
$R$ & $20 \Omega / m$ & $h_{0}$ & 20 \\
$l_{0}$ & $0.37 \mathrm{~m}$ & $h_{2}$ & 0.001 \\
$b_{s}$ & 0.5 & $b_{1}, b_{2}$ & 0.1 \\
$k_{s}$ & $0.0018 \mathrm{Nm} / 1^{\circ}$ & $\Theta$ & -0.055 \\
\hline
\end{tabular}

Note: data from DYNALLOY Inc (2014a,b), Guo et al. (2015) and Elahinia and Ashrafiuon (2002)..

for both configurations. Here, we can see that the use of a second wire generates some disturbances in the main loop, which complicates the control of the system. However, we can also observe a decreased cooling time in the antagonistic case, which speeds up the dynamics of the overall system.

\section{Closed-loop analysis}

A simple controller was applied to both, one SMA wire and two SMA wires actuated system, with the purpose of comparing their closed-loop behavior. A PD control, as the one shown in the Figure 9 (the description of this controller will be given in detail later in the subsection $P D$ control), was applied to both systems for the closed-loop analysis.

Figures 6 and 7 show the results of this test. In the Figure 6 the reference vs the angular position of both configurations is plotted. It is clear that the antagonistic SMA system has a faster response when cooling, due to the control of the overall stiffness of the joint. Here, we can see an average rise time of 1.5 seconds for both cases, while for the fall time there is 3.9 seconds for the single SMA wire vs 2.7 for the two SMA wires, which is an improvement of more than $30 \%$. In addition, the average steady state error of 


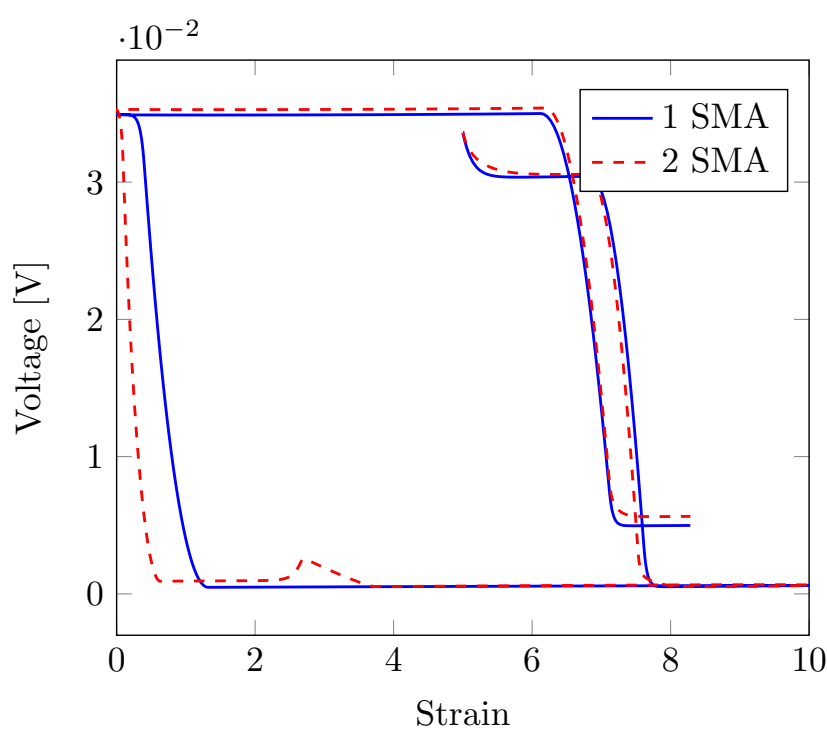

Figure 5. Voltage-Strain hysteresis loop (one biased wire vs antagonistic wires).

$0.296^{\circ}$ for the one wire decreases to $0.124^{\circ}$ with the implementation of the antagonistic configuration.

In spite of this improvement on performance, as mentioned in the last subsection, the use of an antagonistic configuration, increases significantly the nonlinearities of the system, as well as the difficulty for developing a control approach. Nevertheless, the use of the antagonistic configuration brings more advantages than disadvantages and therefore we select the two SMA wires configuration for its faster and more accurate performance. The following section will discuss the performance of 4 different controllers for angular position regulation of the antagonistic configuration system.

\section{Position control}

In this section a controller to regulate the robotic arm's end-effector position is designed. For this, an inner control is selected based on the analysis of four proposed controllers for a joint space control. Afterwards, an overall operational space control is deduced (see Figure 8), which allows regulating the end effector position in the Cartesian coordinate system.

\section{Joint space control: Inner control law}

A joint space control is carried out using the antagonistic configuration of the robotic arm. In order to define an inner control law, four different control approaches are compared with respect to an angular position regulation of the robotic arm.

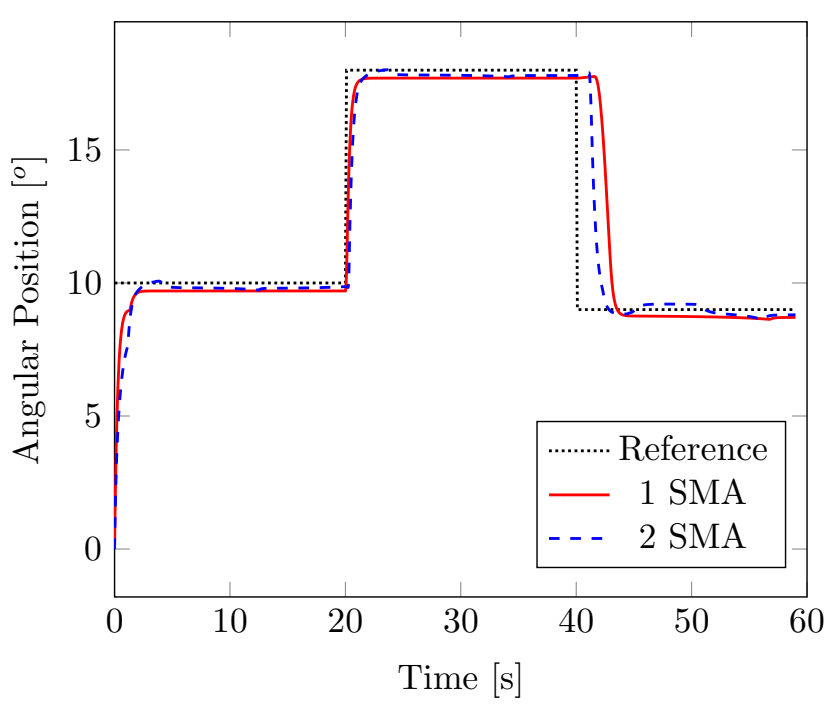

Figure 6. Angular position regulation with $\mathrm{PD}$ control(1 SMA vs 2 SMA).

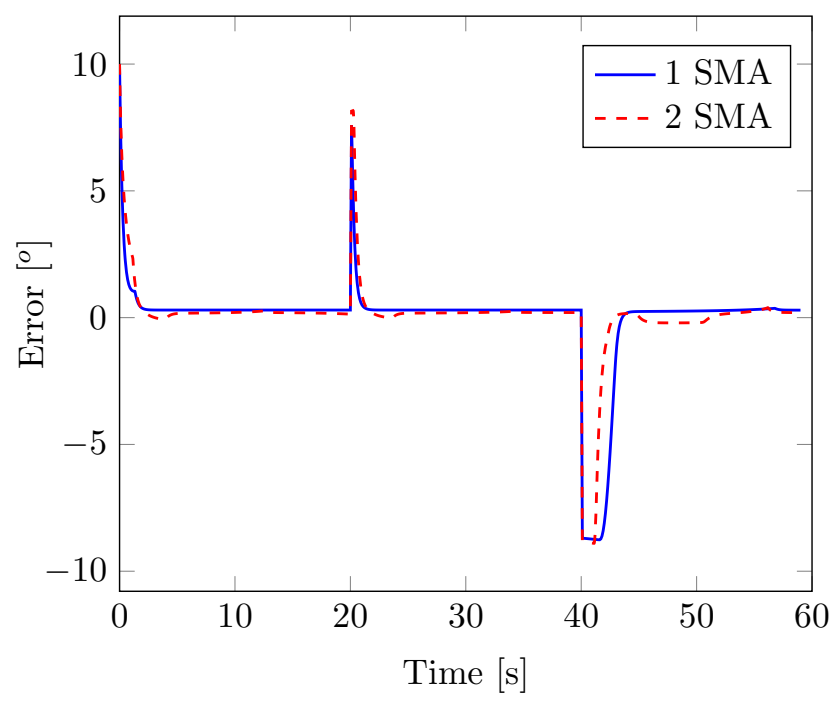

Figure 7. Angular position regulation error with PD control(1 SMA vs 2 SMA).

The controllers selected for this study are: PD control, Sliding Mode Control (SMC), Adaptive Control (AC) and Adaptive Sliding Mode Control (ASMC). The following subsections describe the design and the evaluation of these four controllers.

$P D$ control. For the purpose of comparison we start with the implementation of a PD control, which is one of the simplest approaches for the control of Robotic joint angular position. The advantages and disadvantages of using a PD type of method to control a system with hysteresis is discussed in survey article Hassani et al. (2014). 


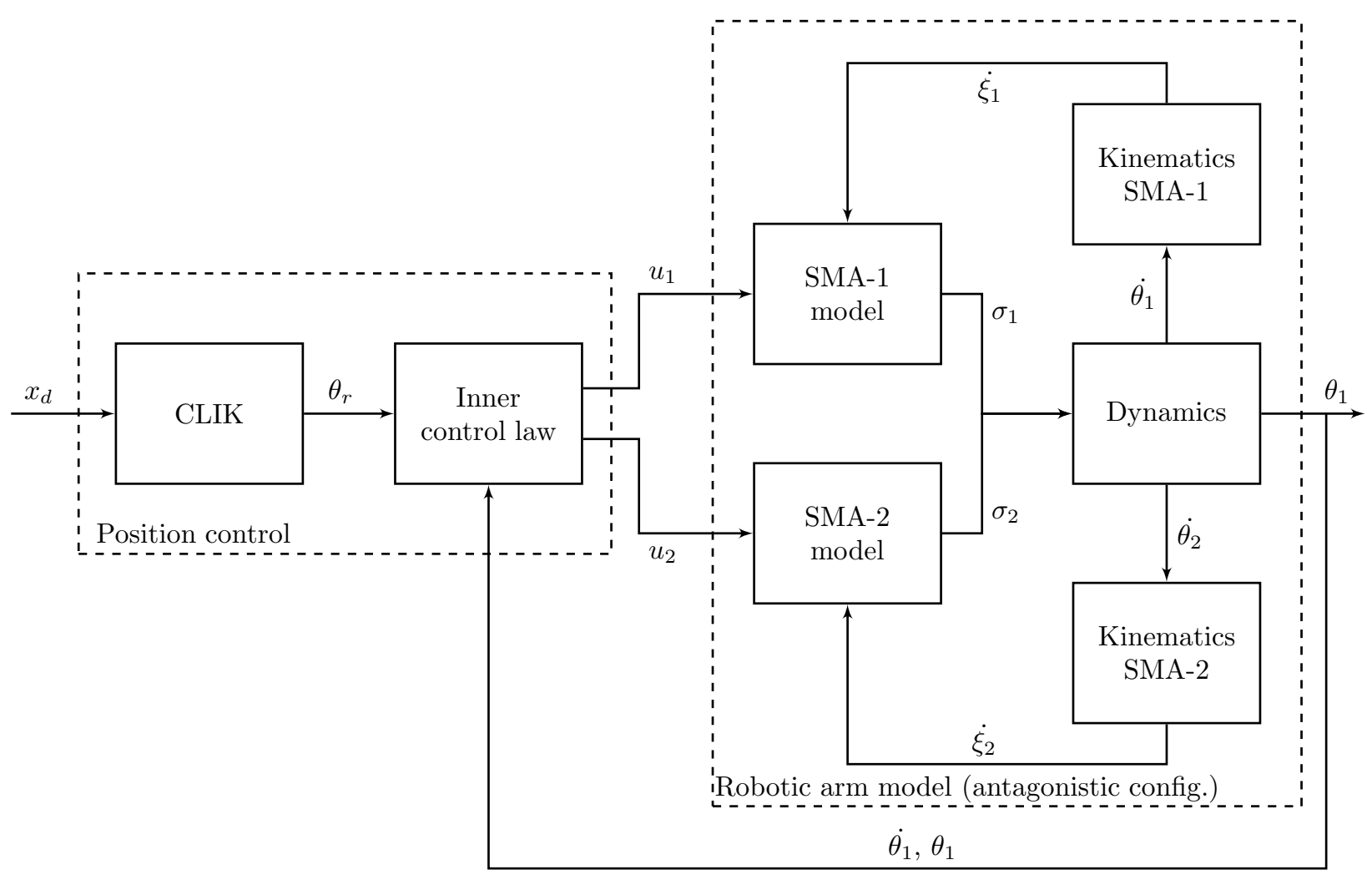

Figure 8. Block diagram of the overall closed-loop system (Operational space control, inner control and SMA actuated robotic arm).

In order to present the four different control approaches, we first define the angular position error (e) as:

$$
e=\theta_{1}-\theta_{r}
$$

where $\theta_{r} \in \mathbb{R}$ is the desired angular position of the arm with respect to the $\mathrm{X}$-axis and $\dot{e}$ is defined as the derivative of the error with respect to the time:

$$
\dot{e}=\frac{d}{d t} e
$$

Then, the control law for the PD control is given by:

$$
u_{i}=k_{p i} e+k_{d i} \dot{e},
$$

where $k_{p i}$ and $k_{d i}$ represent the proportional and derivative gains respectively, and $i=1,2$ for SMA wire 1 and 2. The gains are tuned heuristically and they were set as follows: $k_{p 1}=20, k_{d 1}=6, k_{p 2}=15$ and $k_{d 2}=6$.

In the Figure 9 the complete block diagram for the PD controller is shown. In this diagram, the plant is described by the model presented in the previous sections.

The control signal $u$ is then limited by a saturation block, avoiding voltages over the limit which can

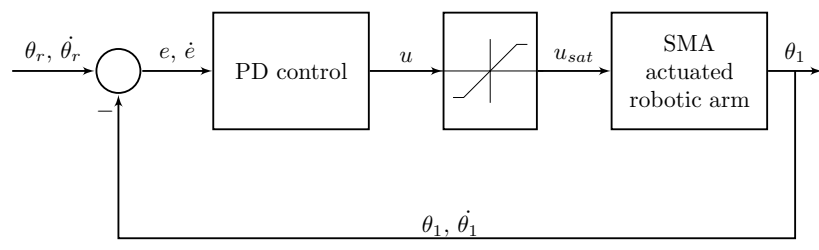

Figure 9. Block diagram for PD Controller.

overheat the wire, thus destroying its shape memory. In the same way, the lower voltage is limited to $0 \mathrm{~V}$ due to the one way heating control inherent to the system. The maximum saturation voltage $\left(V_{H}\right)$ is set for both SMA wire to $V_{H}=10$.

An independent PD control was applied for each wire. The results of this evaluation are shown in the Figures 13 and 14.

The PD control results in an overshoot of $0.163^{\circ}$, a settling time of 2.5 seconds and a steady state error of $0.196^{\circ}$. Although the convenience and fast implementation of this controller is a plus, it is clear that the performance can be improved significantly with different control methods. Furthermore, due to the highly nonlinear behavior of this plant, the 
controller gains obtained for this specific reference will not work as desired if applied to a different input.

Sliding Mode Control. When talking about control of nonlinear systems, the Sliding Model Control (SMC) is one of the most applied strategies due to its robustness and fairly easy design. Here Hassani et al. (2014) would serve as an important survey article. The SMC is a specific type of Variable Structure Control (VSC), which consists of a high-speed switching control law which aims to drive the plant's states onto a userdefined surface (sliding surface). The structure of the control applied will depend on whether the trajectory of the plant is above or below the sliding surface (Liu and Wang, 2011).

The inability of a real actuator to meet the highspeed switching requirements of this type of controller generates a problem known as chattering. This problem is perceived as an oscillation around the sliding surface. To overcome this problem a technique called boundary layer is applied, which is a smooth approximation of the switching element (Young et al., 1999).

The first step to construct a SMC control is to select the sliding surface, which should represent the desired dynamic of the plant's states in steady state. The sliding surface $s$ selected for this case is a firstorder function of the error $e$ (defined in the previous subsection) (Liu and Wang, 2011):

$$
s_{i}=c_{p i} e+c_{d i} \dot{e},
$$

where $c_{p i}$ defines the slope of the sliding surface. Then the control law is established as:

$$
v_{i}=\left\{\begin{array}{l}
M 1_{i} \operatorname{sgn}\left(s_{i}\right),\left|s_{i}\right| \geq \phi_{i} \\
M 2_{i} s_{i},\left|s_{i}\right|<\phi_{i}
\end{array},\right.
$$

where $M 1$ and $M 2$ are definite positive constants, $\phi_{i}$ is the value of the boundary layer and $\operatorname{sgn}(\bullet)$ defines the sign function as:

$$
\operatorname{sgn}(\bullet)=\left\{\begin{aligned}
&-1, \bullet<0 \\
& 0, \bullet=0 \\
& 1, \bullet>0
\end{aligned}\right.
$$

It is important to notice that the voltage is also constrained with identical values as before, avoiding overheating and negative voltages. The block diagram of this controller is shown in the Figure 10.

Using this approach, an independent SMC was applied for each SMA wire. The constant parameters for both controllers were set as follows: The boundary layers $\phi_{1}=10$ degrees, $\phi_{2}=7$ degrees, and limit voltage $V_{1 H}=10 \mathrm{~V}, V_{2 H}=10 \mathrm{~V}$ were chosen, and the gains were tuned heuristically as follows: $c_{p 1}=38$,

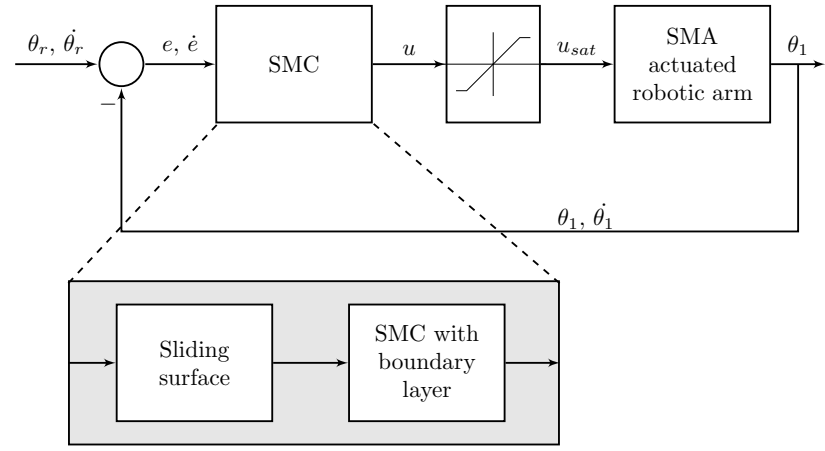

Figure 10. Block diagram for SMC Controller.

$c_{d 1}=25, c_{p 2}=38$ and $c_{d 2}=24$. The results of this evaluation are shown in the Figures 13 and 14. This approach has no overshoot, however, the settling time is 4.2 seconds and the steady state error is $0.13^{\circ}$. In addition, the implementation of this kind of controller can entail inaccuracy due to the hardware limitation in switching speed.

Adaptive Control. This approach includes a set of different techniques which provides a systematic way of automatically adjusting the control parameters in real time, in order to maintain the desired performance while handling parameter and model uncertainties (Landau et al., 2011). The adaptive control techniques have been classified into Direct Adaptive and Indirect Adaptive according to Landau et al. (2011). Similarly Tao (2014) had simply classified the techniques as Adaptive control for Linear Systems or Nonlinear Systems. While similarly the Adaptive techniques can be classified as Adaptive Linear or Adaptive Nonlinear Control.

Different Adaptive control techniques have been applied for the control of SMA wires. For example in the work presented by Kannan et al. (2016b,a) a Direct Linear Adaptive control law is developed for a single SMA wire actuated robotic arm. While in Kannan et al. $(2013,2010)$ an Indirect Adaptive Predictive control using Laguerre functions were used . In Mai et al. (2013) and Pan et al. (2017) Adaptive nonlinear control has been used to control the SMA actuator using universal approximators such as Neural-Networks. An adaptive inverse model was implemented in Mai et al. (2013) using Dynamic Neural Network (DNN) identifier while in Pan et al. (2017) an observer based output feedback control was implemented using Neural-Network in an Indirect Adaptive method. Similarly in Tai and Ahn (2012) a Direct adaptive inverse model based controller using a dynamic neural network was implemented. The Adaptive Nonlinear Control based on universal 
approximators such as Neural-Networks can also be classified under Intelligent Adaptive control. This approach requires the identification of large number of parameters and the quality of approximator depends on the number of neurons and persistent excitation condition. Contrary to these methods, the current paper uses a Direct adaptive control method which requires only one parameter to be tuned in real-time for the control of each SMA.

For the construction of the adaptive control we consider the general dynamic model for the robotic arm presented in equation (9). Extrapolating the work presented in Kannan et al. (2016b,a), the control is designed for an antagonistic actuator. For this, the error given in the equation (13) is considered. Let us define the filtered error $r(t)$ as:

$$
r(t)=\dot{e}(t)+\alpha e(t),
$$

where $\alpha$ is a known positive gain, different for each SMA wire. After algebraic manipulations, the system dynamic in open loop can be mathematically described as (Queiroz et al., 2000):

$$
M(\theta) \dot{r}=-V_{m}(\theta, \dot{\theta}) r+\gamma-\tau
$$

and

$$
\begin{aligned}
\gamma=M(\theta)\left(\ddot{\theta}_{r}+\alpha \dot{e}\right) & +V_{m}(\theta, \dot{\theta})\left(\dot{\theta}_{r}+\alpha e\right) \\
& +g(\theta)+F_{d} \dot{\theta}+\Phi\left(\theta, \theta_{r}\right)
\end{aligned}
$$

Based on the open loop dynamics (20), we choose the control input as:

$$
\tau=\hat{\gamma}+K r,
$$

where $\tau$ is the control input vector, $K$ is a positive control gain matrix and $\hat{\gamma}$ is the estimated of $\gamma$. This value is estimated as follows:

$$
\hat{\gamma}=\Gamma^{-1} r
$$

where $\Gamma$ is the positive adaptation gain. Finally the closed-loop dynamic is given by:

$$
M(\theta) \dot{r}=-V_{m}(\theta, \dot{\theta}) r+K r+\tilde{\gamma}
$$

and $\tilde{\gamma}=\gamma-\hat{\gamma}$.

The gains were set as follows: $\alpha_{1}=0.9, K_{1}=19$, $\alpha_{2}=0.85$ and $K_{2}=13$. The Figure 11 shows the block diagram of the closed-loop system with the adaptive control. This controller achieved a steady state error of $0.022^{\circ}$ with a $2.7 \mathrm{sec}$ settling time, moreover a practically non-existent overshoot of $0.077^{\circ}$. The results of this evaluation are shown in the Figures 13 and 14.

The adaptive control has an excellent performance considering the nonlinear behavior of the system.

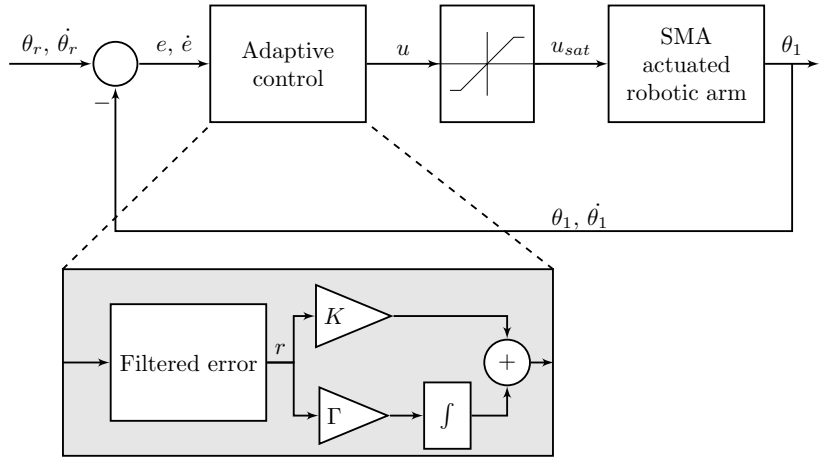

Figure 11. Block diagram for Adaptive Controller.

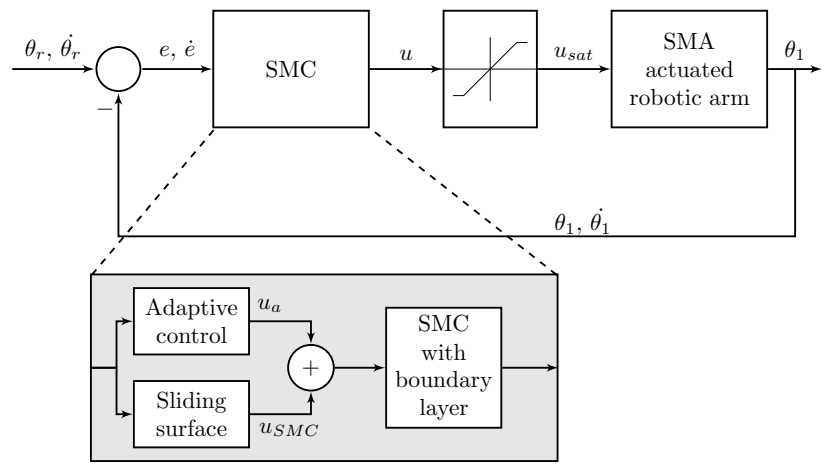

Figure 12. Block diagram for ASMC Controller.

Adaptive Sliding Mode Control. The Adaptive Sliding Mode Control (ASMC) combines the formulation of SMC and adaptive control. In Hassani et al. (2014) the different types of sliding mode control methods have been discussed in the context of hysteretic systems. Here the SMC is developed and an adaptive term is included in the control law as follows:

$$
u_{i}=\left\{\begin{array}{l}
M 1_{i} \operatorname{sgn}\left(s_{i}\right),\left|s_{i}\right| \geq \phi_{i} \\
M 2_{i} s_{i}+\tau,\left|s_{i}\right|<\phi_{i}
\end{array},\right.
$$

and $\tau$ is defined as in the equation (22). This approach behaves as a SMC when the states are further than the boundary layer from the sliding surface $\left(\left|s_{i}\right| \geq \phi_{i}\right)$. On the other hand, when the states are inside the boundary layer, the control law switches to include an adaptive term $\left(u_{a}\right)$ and the original control term from the SMC configuration $\left(u_{s m c}\right)$.

The block diagram is shown in the Figure 12. The settings used for the combination of both, the SMC and the adaptive part, were tuned differently from the independent approach of each one. This procedure allows obtaining the best performance with the current ASMC approach. In spite of the efforts tuning the system, the performance was not as expected. For the SMA-1 the gains are $\alpha_{1}=1, K_{1}=2, c_{p 1}=10$, 
$c_{d 1}=2.5$, while for the SMA- $2 \alpha_{2}=0.8$ and $K_{2}=1.3$, $c_{p 2}=10$ and $c_{d 2}=3$. The boundary layer and limit voltages where set as in the SMC controller. The maximum overshoot was $0.394^{\circ}$, with a settling time of $4 \mathrm{sec}$ and a steady state error of $0.164^{\circ}$. A disadvantage of this approach is the complicated tuning, due to the multiple control gains.

Results discussion. For a quantitative comparison, the 4 controllers presented above are analyzed by evaluating three different characteristics: maximum overshoot (OS), average settling time (ST), and average steady state error (SSE). Table 2 summarizes these characteristics, allowing to evaluate the performance of the four different controllers. Here we can see that although the smallest settling time is achieved with the PD control, it has the highest steady state error. The SMC is ideal to avoid overshoot, nonetheless, has the highest settling time.

In an overall view, the ASMC has the worst results of all four, with a high settling time and the highest overshoot. Thereupon, the controller with the best performance for this specific system turned out to be the adaptive control with the second best settling time and a really low steady state error, in addition to the small overshoot of $0.077^{\circ}$. Figures 13 and 14 show the comparative graphs for the performance in position regulation of the four controllers.

Table 2. Comparative table of controller performance.

\begin{tabular}{llll}
\hline Control & OS $\left[^{\circ}\right]$ & ST $[\mathrm{s}]$ & SSE $\left[^{\circ}\right]$ \\
\hline PD & 0.163 & 2.5 & 0.196 \\
SMC & 0 & 4.2 & 0.13 \\
Adaptive & 0.077 & 2.7 & 0.022 \\
ASMC & 0.394 & 4 & 0.164 \\
\hline
\end{tabular}

OS: Overshoot percentage; ST: Settling time; SSE: Steady State Error.

In order to ensure that the controller is capable of dealing with changes in the systems parameters, due to environmental conditions, the adaptive approach is selected to perform a disturbance test. This disturbance test is useful for emulating the repeatability problem presented in the SMA actuators. This test is discussed in the next subsection.

\section{Disturbance test}

One of the main concerns when dealing with control of SMA wires, in addition to the inherent characteristics of SMA materials, is the susceptibility of this actuator to thermal disturbances. Ambient temperature variations or the presence of wind, can significantly affect the performance of the system. For

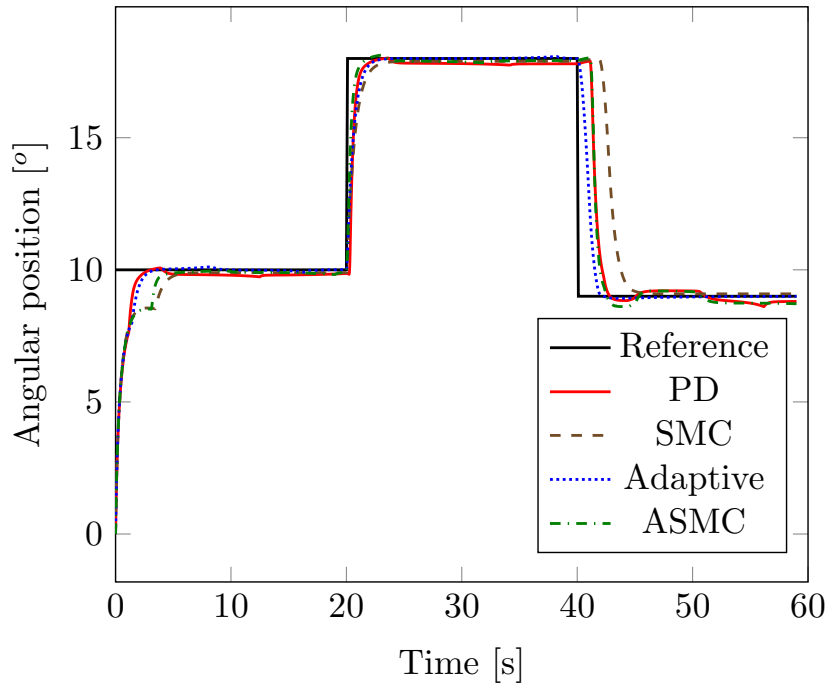

Figure 13. Comparison of four controllers for angular position regulation.

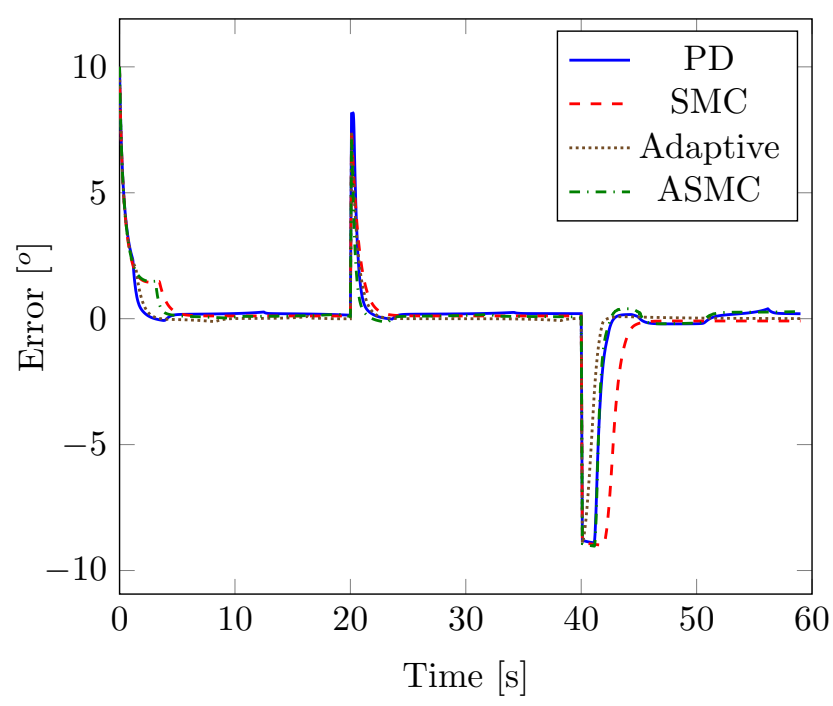

Figure 14. Comparison of position error of four controllers.

this reason, a disturbance rejection test was performed to the selected adaptive control approach.

This test was performed by simulating multiple wind gusts at different air flow rates over the wire by means of changing the wire's convection coefficient $(h)$. The correspondent values of $h$ for different air flow rates were taken from the study present by Pathak et al. (2008), and can be seen in the Table 3.

The disturbance was modeled as a 2 seconds step signal over the $h$ coefficient, repeating at 13,30 and 45 seconds. Four different air flow rates were considered (Table 3). The results of this test are shown in Figure 15 , and it is important to mention that the gains for the controller are the same as in the previous section. 
Table 3. Effect of air flow rate at $22{ }^{\circ} \mathrm{C}$ on the convection coefficient for a $0.25 \mathrm{~mm}$ diameter SMA wire.

\begin{tabular}{ll}
\hline Flow rate $[\mathrm{m} / \mathrm{s}]$ & $\mathrm{h}\left[\mathrm{W} / \mathrm{m}^{2} \mathrm{~K}\right]$ \\
\hline 0 & 120 \\
0.7 & 230 \\
1.2 & 380 \\
2.25 & 430 \\
3.1 & 490 \\
\hline
\end{tabular}

Note: Data from study presented in Pathak et al. (2008).

In Figure 15(a) we can observe the complete simulation with disturbances at 13, 30 and 45 seconds at different air flow rates. In addition, Figure 15 (b) to (d) present a closer look at the time of the disturbance, where the effect of the thermal disturbance is evident, we can observe a maximum deviation of $0.2^{\circ}$ from reference in the worst case scenario (air flow rate of 3.1 $\mathrm{m} / \mathrm{s}$ ). The effect of the disturbance is easily observed in Figure 16, where changes on SMA-1 temperature $\left(T_{1}\right)$ due to presence of disturbances, are shown. Figure 16(a) shows the complete simulation, where the thermal disturbance is really noticeable compared to the thermal dynamic of the non perturbed system. This can also be observed in Figure 17 which shows the voltage of the SMA-1 $\left(V_{1}\right)$. In Figures 17(b) and (c) the controller reacts to the disturbance by increasing the voltage to compensate the fast cooling induced by the forced air convection. For the disturbance at 45 seconds (Figure 17(d)) there is no response from the controller thus it is already at the lowest voltage (0 $\mathrm{V})$.

From this test we can conclude that the selected adaptive control is capable of dealing with one of the most common disturbances affecting SMA wires (thermal disturbance). The adaptive approach is then used for the design of the operational space control, discussed in the next section.

\section{Operational space control: Position regulation}

The operational space is a framework used to analyze the system from the end-effector's dynamic behavior (Khatib, 1987). Based on this approach the development of an operational space position control will be discussed in this section. The control law presented here is decomposed into two separate controllers. First, the inner control law that works in the joint space, which regulates the output angle of the actuator $\theta_{1}$. Second part is the Closed Loop Inverse Kinematics (CLIK) algorithm in the outer loop which generates the required joint space reference necessary to control the position of the end-effector (a)

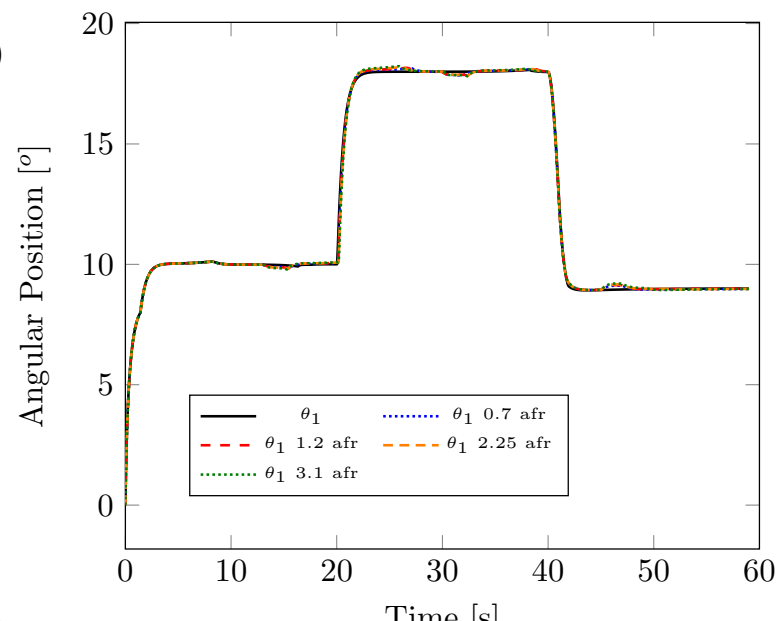

(b)

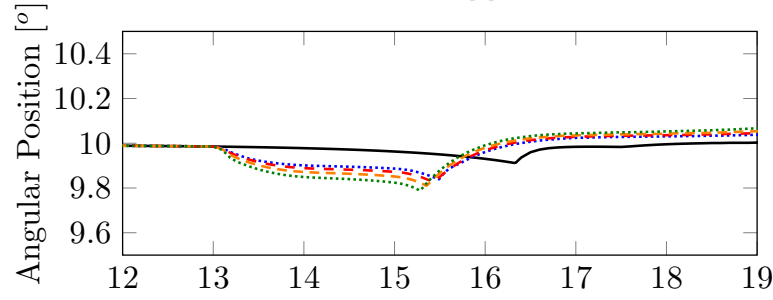

(c)

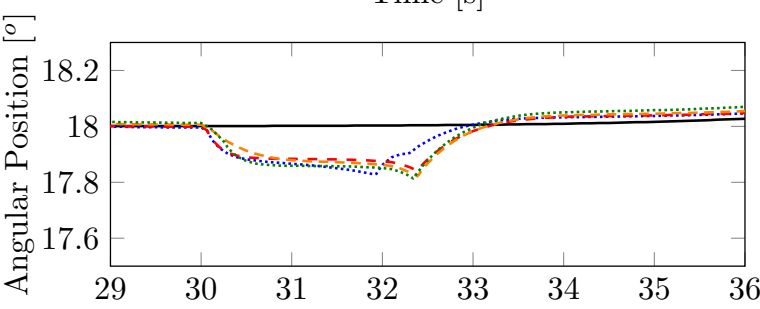

(d)

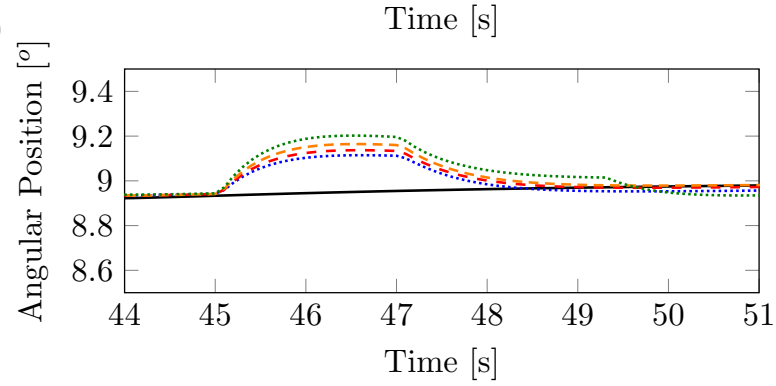

Figure 15. Angular position with disturbance at different instants and air flow rates using adaptive control at (a) full simulation, (b) 13 seconds, (c) 30 seconds and (d) 45 seconds.

afr: air flow rate $[\mathrm{m} / \mathrm{s}]$; Trans. Temps.: Transformations temperatures.

in the Cartesian space. The control law and the CLIK algorithm are explained in further detail in the following subsections.

Inner Control Law. The inner control law regulates the rotational movement of the end effector. The controlled variable is the angular position of coupler-1 $\left(\theta_{1}\right)$ (see Figure 1). For the inner control, an adaptive controller 
(a)

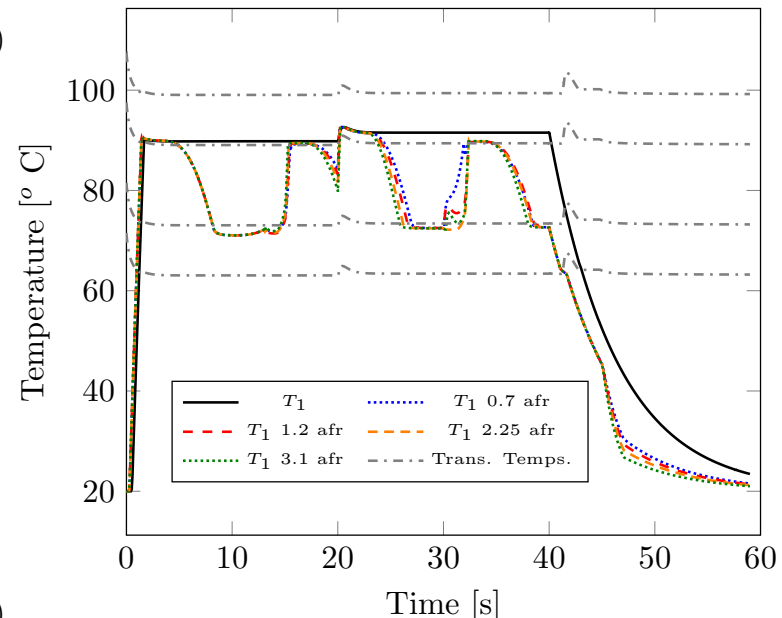

(b)

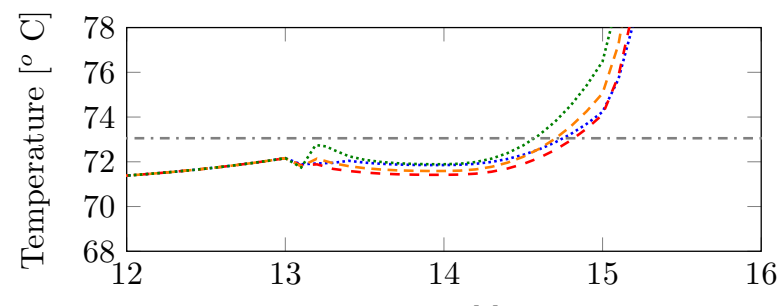

(c)

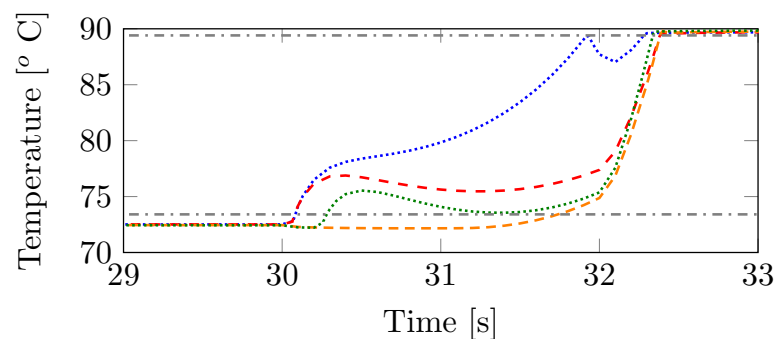

(d)

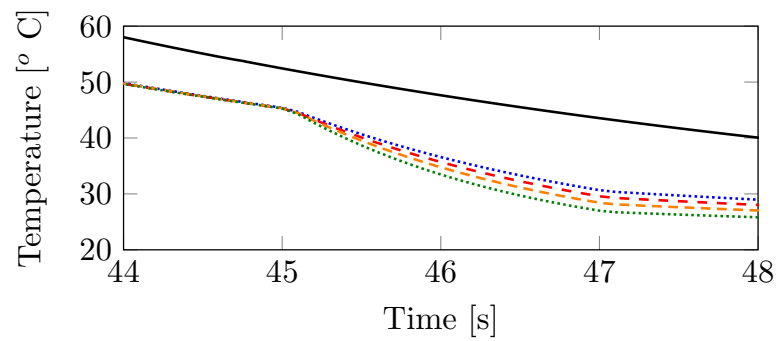

Figure 16. SMA-1 Temperature with disturbance at different instants and air flow rates using adaptive control at (a) full simulation, (b) 13 seconds, (c) 30 seconds and (d) 45 seconds.

afr: air flow rate $[\mathrm{m} / \mathrm{s}]$

was chosen based on the results of the comparison previously discussed. The basic control law is given by the equation (22). This control law is applied in an inner closed-loop as shown in the Figure 8.

Closed Loop Inverse Kinematics (CLIK). The CLIK algorithm is in the outer loop as seen in the Figure 8. The CLIK algorithm uses as input the references (a)

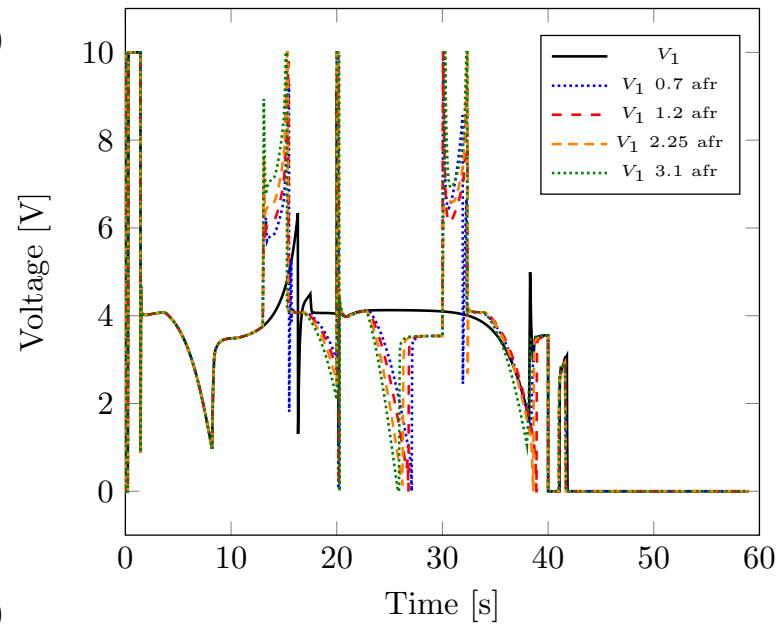

(b)
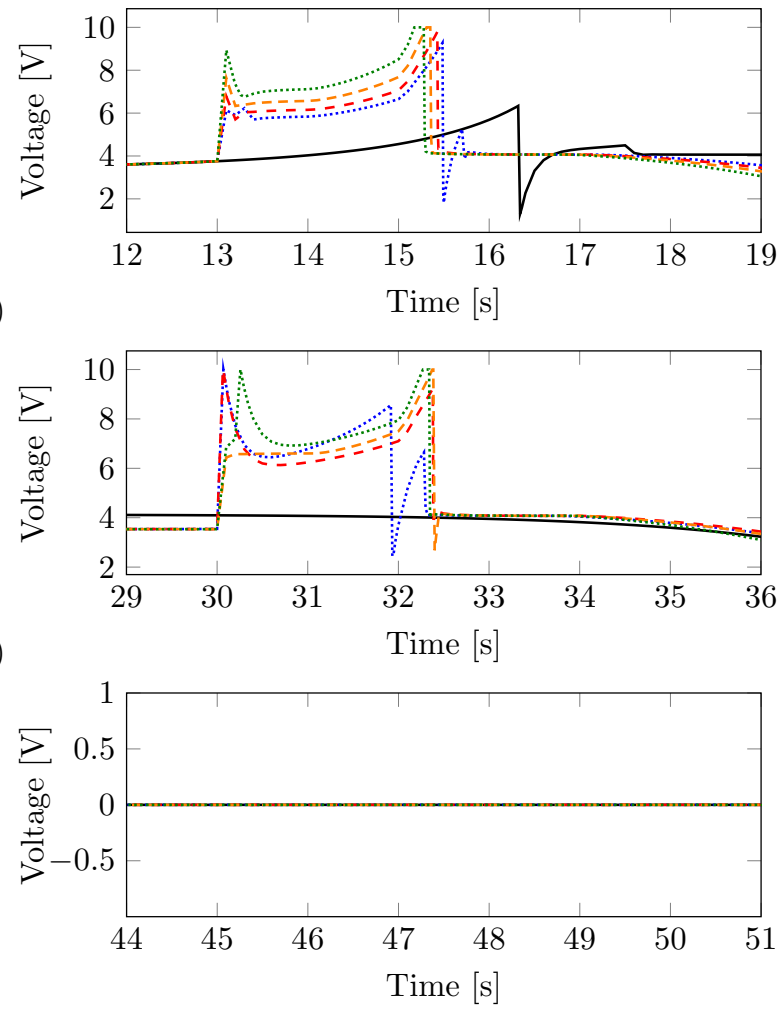

Figure 17. $V_{1}$ with disturbance at different instants and air flow rates using adaptive control at (a) full simulation, (b) 13 seconds, (c) 30 seconds and (d) 45 seconds. afr: air flow rate $[\mathrm{m} / \mathrm{s}]$.

in a Cartesian coordinate system and gives as output the reference in the joint space for the inner control. The schematic diagram of the CLIK algorithm is illustrated in Figure 18. This is described by the following equation (Khatib, 1987):

$$
\dot{q}=J_{A}^{T}(q) K_{r} e_{o s}
$$




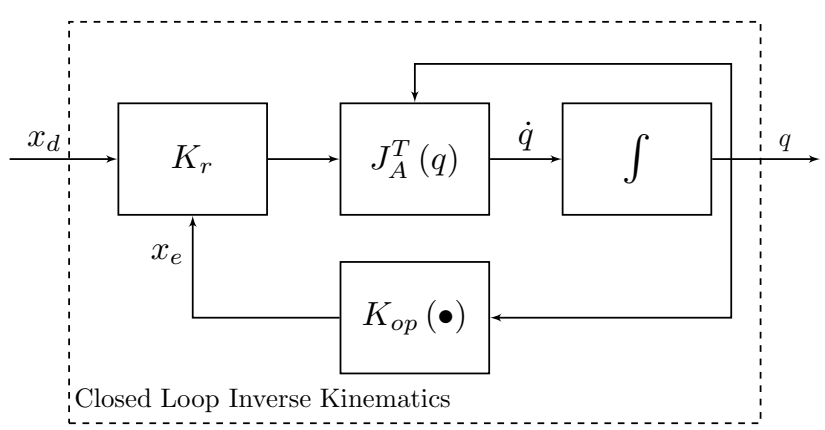

Figure 18. Closed Loop Inverse Kinematics block diagram.

where $\dot{q}$ is the derivative of the state vector with respect to the time, $J_{A}$ is the analytical Jacobian of the robotic arm, $K_{r} \in \mathbb{R}^{n}$ is a symmetric gain matrix, $n$ is the number of states in the dynamic model and $e_{o s}$ is the operational space error defined as:

$$
e_{o s}=x_{d}-x_{e}
$$

herein, $x_{d}$ is the set of Cartesian coordinates for the end effector's desired position and $x_{e}$ is the end effector's position vector. This control represents a simple proportional control, which takes into account the direct and inverse dynamics of the one DOF robot arm. Equation (28) shows the analytical Jacobian of the robotic system and the equation (29) describes the direct kinematics as follows:

$$
\begin{gathered}
J_{A}(q)=\frac{\partial K_{o p}(\cdot)}{\partial q}=\left[\begin{array}{c}
-a_{1} \sin \left(q_{1}\right) \\
-a_{1} \cos \left(q_{1}\right)
\end{array}\right] \\
K_{o p}(\cdot)=\left[\begin{array}{c}
a_{1} \cos \left(q_{1}\right) \\
a_{1} \sin \left(q_{1}\right)-h
\end{array}\right]
\end{gathered}
$$

where $a_{1}$ is the length of the second link (150 mm), $h$ is the length of the first link $(100 \mathrm{~mm})$ plus the base height $(50 \mathrm{~mm})$ and we define $q=\theta_{1}$.

The open and closed loop performance of the SMA actuated robotic arm was evaluated through simulations using Simulink/MATLAB. The open-loop test was performed by applying the maximum safe voltage $\left(V_{1 H}\right)$ to the SMA-1 while no voltage was applied to SMA-2 and then vice versa. The results of this simulation are shown in the Figure 19. This figure shows the major hysteresis loop (Temperature Strain).

The plot of the hysteresis curve shows a double hysteresis loop, however we can assume a big difference in size between the two loops. This phenomenon is due to the use of 2 SMA wires configuration, called as antagonistic configuration. Nonetheless, this system is not an antagonistic application in the common sense, since the second wire does not really actuate the

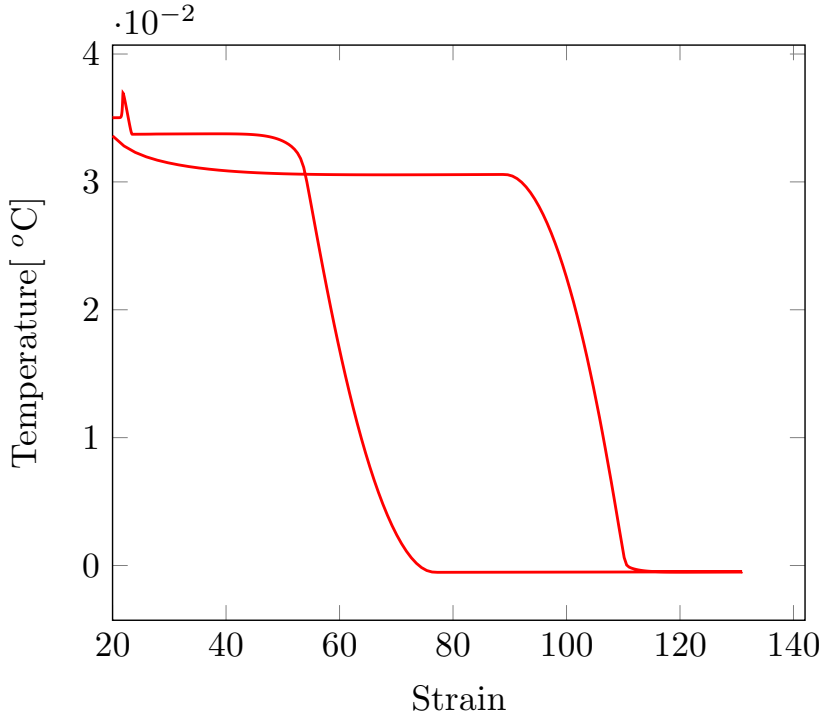

Figure 19. Open-loop system hysteresis curve Temperature Strain.

end-effector, but just permits to adjust the overall stiffness of the joint.

In order to carry out a closed-loop analysis, a position regulation was performed. The step response test was used with a sequence of 3 steps apart by 20 seconds with an amplitude computed by equation (30). This is shown in Figure 20 (solid line).

$$
x_{d}=\left[\begin{array}{c}
a_{1} \cos (N(i) \pi / 180) \\
a_{1} \sin (N(i) \pi / 180)-h
\end{array}\right]
$$

where $N=[10,18,9]$. The origin of the system is set at the center of the upper face of the robotic arm's base.

The Closed Loop Inverse Kinematics gain $\left(K_{r}\right)$ was set to 300 , in order to achieve a fast response through this controller. The gains for the adaptive control were set as follows: $\alpha_{1}=0.9, \alpha_{2}=0.8, K_{1}=19, K_{2}=13$, and voltage limits $V_{1 H}=10 \mathrm{~V}, V_{2 H}=10 \mathrm{~V}$. The results of this simulation are shown in the Figures 20 to 21 .

In the Figure 20 it can be seen that the overshoot is almost nonexistent with a maximum positive overshoot of $0.3 \%$ and $0.156 \%$ for axis $X$ and $Z$ respectively, and $0.03 \%$ and $2.39 \%$ for the negative one in each axis. This is attributed to the derivative effect in the filtered error. In addition, the maximum steady state error in the $X$-axis was $0.002 \%$ and $0.006 \%$ for the $Z$-axis. Figure 21 shows the error norm of the system, with maximum value of 0.0284 during the biggest down step. Furthermore, the system presents an average settling time of 4.2 seconds.

The SMA wires' temperatures and transformation temperatures are shown in the Figure 22, where 

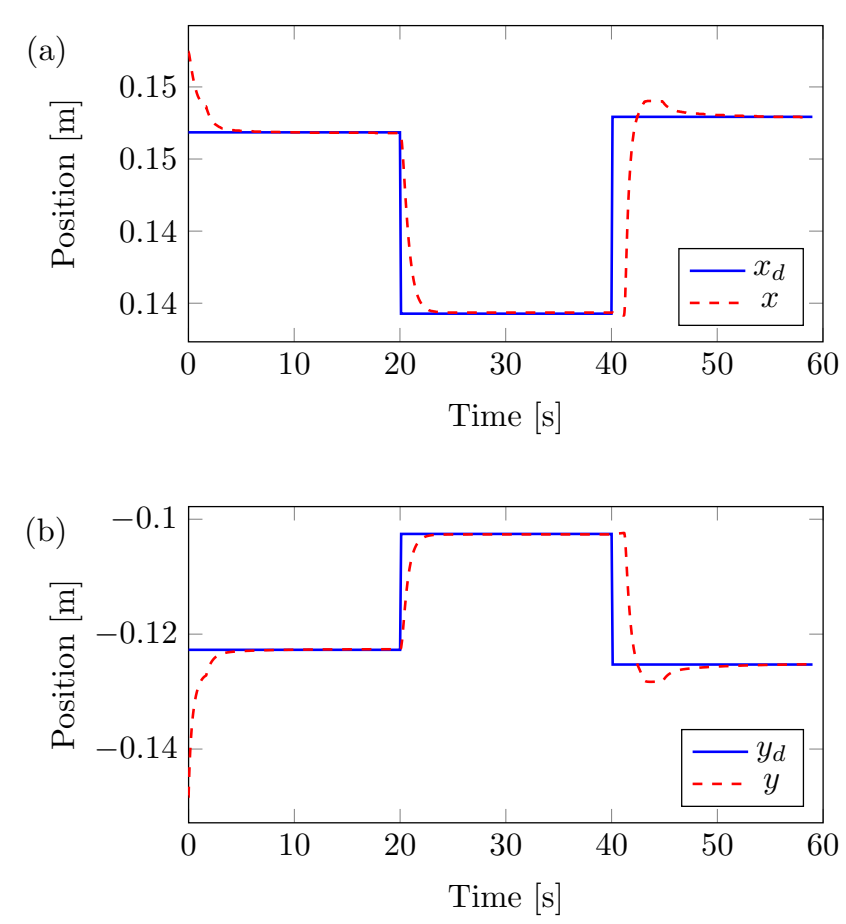

Figure 20. Operational space control for position regulation step response (a) $\mathrm{X}$ axis (b) $\mathrm{Z}$ axis.

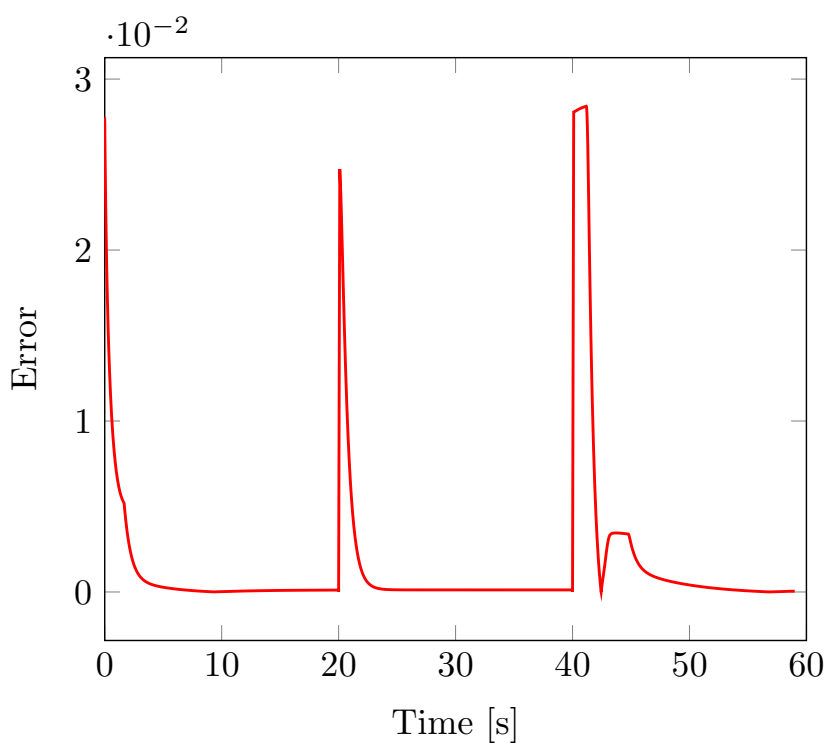

Figure 21. Step response error norm.

we can observe the influence of SMA-2 over the cooling dynamic of the system from $t=40$ seconds. When SMA-2 reaches the austenite transformation temperature it causes an increment on the stress, which at the same time leads to a rise in the transformation temperatures of SMA-1. This effect simultaneously leads to an accelerated inverse (a)

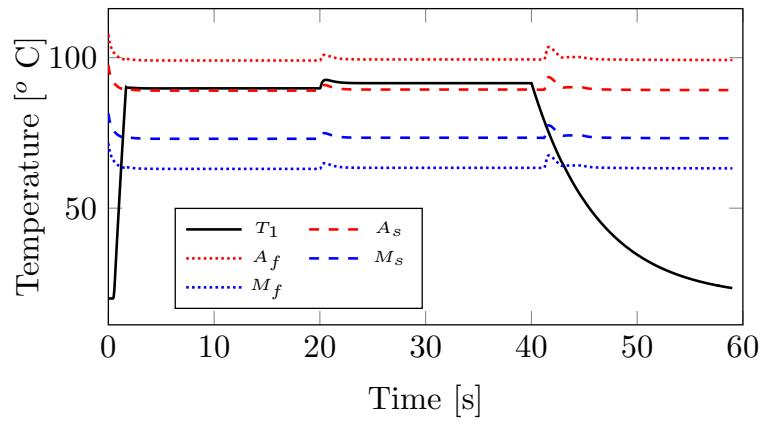

(b)

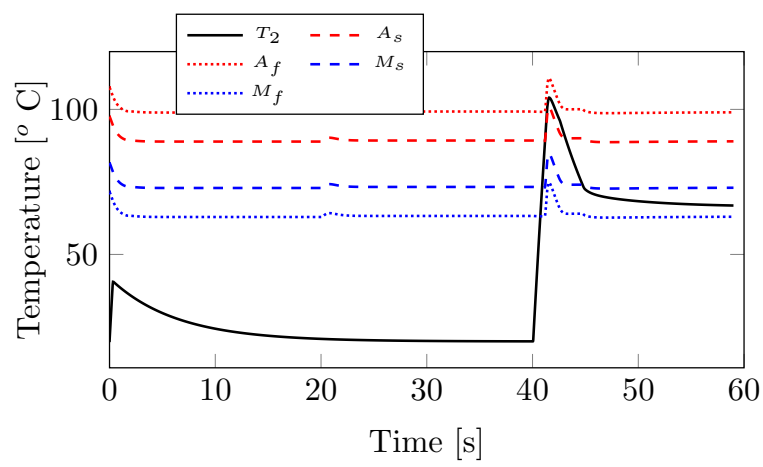

Figure 22. Temperature vs Transformation temperatures, (a) SMA-1 (b) SMA-2

transformation austenite to martensite, allowing the system to reach the lower reference faster.

Figure 23 illustrates the control signals applied to the system during the closed-loop test. The control signal is given in Volts and it is limited to avoid thermal damage to the SMA wires; as an excess voltage can destroy its memory effect. The gains of the system are tuned heuristically to achieve a faster and more accurate response. Table 4 shows the gains used for the adaptive controllers to regulate each SMA wire. The gains of the SMA-2 are noticeably smaller than those of the SMA-1, since the SMA-2 adjusts the stiffness of the joint. This means that the SMA-2 does not actuate directly the end-effector, thus its rate of response is not as critical as SMA-1.

Table 4. Adaptive Control gains.

\begin{tabular}{llll}
\hline Wire & $K$ & $\Gamma$ & $\alpha$ \\
\hline SMA-1 & 19 & 5 & 0.9 \\
SMA-2 & 13 & 2.5 & 0.85 \\
\hline
\end{tabular}

\section{Conclusions}

In this paper we have presented a SMA wire actuated lightweight robotic arm, which is intended to be an alternative to flying manipulators designs. This arm 


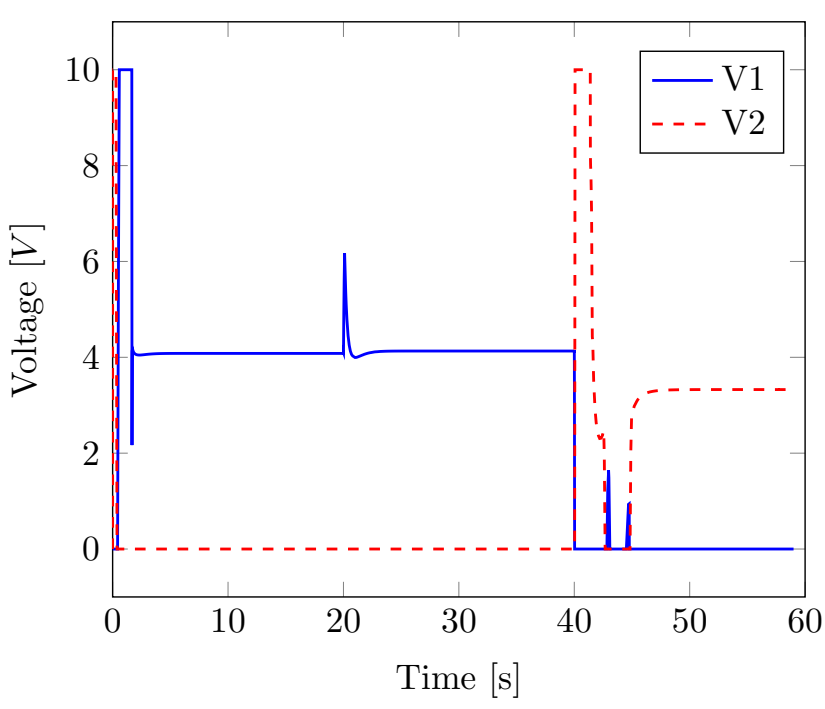

Figure 23. Voltage input.

was actuated by a couple of antagonistic SMA wires. It has a total weight of $48 \mathrm{~g}$ and a range of movement up to 85 degrees on the $X-Z$ plane.

A comparison between one biased wire vs an antagonistic system (2 SMA wires) was carried out in open and closed-loop. During this analysis, it became clear that the use of an antagonistic system accelerates the response of the system and increases the accuracy. Following this analysis, a comparison among 4 different controllers for the antagonistic system was developed. Different control approaches such as PD, SMC, Adaptive Control and ASMC were tested. As a result of the comparative analysis, it was concluded that the best performance was achieved by the adaptive control law. Finally an operational space control was developed based on the closed loop inverse dynamics of the arm presented.

Future work will be the construction and experimental test of the presented design. In addition, the proposed SMA wires based robotic arm will be assembled as flying manipulator and attached to a small quadcopter.

\section{Declaration of conflicting interests}

The author(s) declared no potential conflicts of interest with respect to the research, authorship and/or publication of this article

\section{Funding}

This research received no specific grant from any funding agency in the public, commercial, or not-for-profit sectors.

\section{References}

Andani MT, Anderson W and Elahinia M (2015) Design, modeling and experimental evaluation of a minimally invasive cage for spinal fusion surgery utilizing superelastic nitinol hinges. Journal of intelligent material systems and structures 26(6): 631-638.

Barbarino S, Ameduri S, Lecce L and Concilio A (2009) Wing shape control through an sma-based device. Journal of intelligent material systems and structures 20(3): 283-296.

Bellicoso CD, Buonocore LR, Lippiello V and Siciliano B (2015) Design, modeling and control of a 5-dof lightweight robot arm for aerial manipulation. 2015 23rd Mediterranean Conference on Control and Automation, MED 2015 - Conference Proceedings : 853-858.

Colorado J, Barrientos A and Rossi C (2011) Músculos inteligentes en robots biológicamente inspirados: Modelado, control y actuación. Revista Iberoamericana de Automática e Informática Industrial RIAI 8(4): 385 $-396$.

DYNALLOY Inc (2014a) Flexinol actuator wire technical and design data. Available at: http://www.dynalloy.com/tech_data_wire.php. (accessed 26 April 2016).

DYNALLOY Inc (2014b) Technical characteristics of flexinol. Available at: http://www . dynalloy.com/pdfs/TCF1140.pdf. (accessed 26 April 2016).

Elahinia MH and Ashrafiuon H (2002) Nonlinear control of a shape memory alloy actuated manipulator. Journal of Vibration and Acoustics 124(4): 566-575.

Gao F, Wang Z, Wang Y, Wang Y and Li J (2014) A prototype of a biomimetic mantle jet propeller inspired by cuttlefish actuated by sma wires and a theoretical model for its jet thrust. Journal of Bionic Engineering 11(3): 412-422.

Guo Z, Pan Y, Wee LB and Yu H (2015) Design and control of a novel compliant differential shape memory alloy actuator. Sensors and Actuators A: Physical 225: 7180.

Hassani V, Tjahjowidodo T and Do TN (2014) A survey on hysteresis modeling, identification and control. Mechanical Systems and Signal Processing 49(12): 209 -233 .

Hulea M and Caruntu CF (2014) Spiking neural network for controlling the artificial muscles of a humanoid robotic arm. In: 2014 18th International Conference on System Theory, Control and Computing (ICSTCC). IEEE, pp. 163-168.

Jafari J, Zebarjad S and Sajjadi S (2008) Effect of prestrain on microstructure of niti orthodontic archwires. Materials Science and Engineering A 473(12): 42-48. 
Kannan S (2011) Modélisation et Commande dActionneurs à Alliage à Mémoire de Forme. PhD Thesis, l'École Nationale Supérieure d'Arts et Métiers.

Kannan S, Bezzaoucha S, Quintanar-Guzmán S, OlivaresMendez MA and Voos H (2016a) Adaptive control of robotic arm with hysteretic joint. In: Proceedings of the 4th International Conference on Control, Mechatronics and Automation, ICCMA '16. Barcelona: ACM, pp. 4650.

Kannan S, Giraud-Audine C and Patoor E (2010) Laguerre model based adaptive control of antagonistic shape memory alloy (sma) actuator. In: Proc. SPIE 7643, Active and Passive Smart Structures and Integrated Systems 2010, volume 7643. pp. 764307-764307-12.

Kannan S, Giraud-Audine C and Patoor E (2013) Application of laguerre based adaptive predictive control to shape memory alloy (sma) actuator. ISA Transactions 52(4): 469 - 479.

Kannan S, Quintanar-Guzmán S, Bezzaoucha S, OlivaresMendez MA and Voos H (2016b) Adaptive control of hysteretic robotic arm in operational space. In: Proceedings of the 5th International Conference on Mechatronics and Control Engineering, ICMCE '16. Venice: ACM, pp. 92-96.

Kennedy DK, Straub FK, Schetky LM, Chaudhry Z and Roznoy R (2004) Development of an sma actuator for in-flight rotor blade tracking. Journal of intelligent material systems and structures 15(4): 235-248.

Khatib O (1987) A unified approach for motion and force control of robot manipulators: The operational space formulation. IEEE Journal on Robotics and Automation 3(1): 43-53.

Khodayari A, Talari M and Kheirikhah MM (2011) Fuzzy pid controller design for artificial finger based sma actuators. In: 2011 IEEE International Conference on Fuzzy Systems (FUZZ-IEEE 2011). pp. 727-732.

Ko J, Jun MB, Gilardi G, Haslam E and Park EJ (2011) Fuzzy pwm-pid control of cocontracting antagonistic shape memory alloy muscle pairs in an artificial finger. Mechatronics 21(7): 1190-1202.

Landau ID, Lozano R, M'Saad M and Karimi A (2011) Adaptive control: algorithms, analysis and applications. Springer Science \& Business Media.

Liang C and Rogers CA (1990) One-dimensional thermomechanical constitutive relations for shape memory materials. Journal of intelligent material systems and structures 1(2): 207-234.

Liu J and Wang X (2011) Advanced Sliding Mode Control for Mechanical Systems: Design, Analysis and MATLAB Simulation. Springer-Verlag Berlin Heidelberg.
Mai H, Song G and Liao X (2013) Adaptive online inverse control of a shape memory alloy wire actuator using a dynamic neural network. Smart Materials and Structures 22(1): 015001.

Mataee MG, Andani MT and Elahinia M (2015) Adaptive anklefoot orthoses based on superelasticity of shape memory alloys. Journal of intelligent material systems and structures 26(6): 639-651.

Moghaddam NS, Skoracki R, Miller M, Elahinia M and Dean D (2016) Three dimensional printing of stiffness-tuned, nitinol skeletal fixation hardware with an example of mandibular segmental defect repair. Procedia CIRP 49: 45-50.

Nematzadeh F and Sadrnezhaad S (2012) Effects of material properties on mechanical performance of nitinol stent designed for femoral artery: Finite element analysis. Scientia Iranica 19(6): 1564 - 1571.

Nespoli A, Dallolio V, Villa E and Passaretti F (2015) A new design of a nitinol ring-like wire for suturing in deep surgical field. Materials Science and Engineering C 56: 30-36.

Pan Y, Guo Z, Li X and Yu H (2017) Output-feedback adaptive neural control of a compliant differential sma actuator. IEEE Transactions on Control Systems Technology PP(99): 1-9.

Pandis N and Bourauel CP (2010) Nickel-titanium (niti) arch wires: the clinical significance of super elasticity. Seminars in Orthodontics 16(4): 249-257. Latest Controversies in Orthodontics: From in Vitro Data to in Vivo Evidence.

Pathak A, Brei D and Luntz J (2008) Experimental characterization of the convective heat transfer from shape memory alloy (sma) wire to various ambient environments. In: Student's papers. Ascona, Switzerland: Pathak,A.; Mechanical Engineering, University of Michigan, 2250 GG Brown, Ann Arbor, MI 48109-2126, USA, apathak@umich.edu, p. 12 pp.

Queiroz MS, Dawson DM, Nagarkatti SP and Zhang F (2000) Lyapunov-Based Control of Mechanical Systems. Springer.

Quintanar-Guzmán S, Kannan S, Olivares-Mendez MA and Voos H (2016) Operational space control of a lightweight robotic arm actuated by shape memory alloy (sma) wires. In: ASME 2016 Conference on Smart Materials, Adaptive Structures and Intelligent Systems, volume Volume 2: Modeling, Simulation and Control; Bio-Inspired Smart Materials and Systems; Energy Harvesting. Stowe, Vermont: American Society of Mechanical Engineers.

Quintanar-Guzmán S, Reyes-Reyes J and Arellano-Sánchez Mdc (2014) Modelado y control de un sistema electrotérmico-mecánico móvil basado en alambres 
musculares. In: XVI Congreso Latinoamericano de Control Automático, CLCA 2014. Asociación de México de Control Automático, pp. 834-839.

Rao A, Srinivasa AR and Reddy JN (2015) Design of Shape Memory Alloy (SMA) Actuators. SpringerBriefs in Computational Mechanics. Springer International Publishing.

Rodrigue H, Cho S, Han MW, Bhandari B, Shim JE and Ahn SH (2016) Effect of twist morphing wing segment on aerodynamic performance of uav. Journal of Mechanical Science and Technology 30(1): 229-236.

Shin BH, Lee KM and Lee SY (2015) A miniaturized tadpole robot using an electromagnetic oscillatory actuator. Journal of Bionic Engineering 12(1): 29-36.

Son HM, Kim MY and Lee YJ (2009) Tunable-focus liquid lens system controlled by antagonistic winding-type sma actuator. Optics Express 17(16): 14339-14350.

Sreekumar M, Nagarajan T and Singaperumal M (2009) Application of trained niti sma actuators in a spatial compliant mechanism: Experimental investigations. Materials \& Design 30(8): 3020-3029.

Tai NT and Ahn KK (2012) Output feedback direct adaptive controller for a sma actuator with a kalman filter. IEEE Transactions on Control Systems Technology 20(4): 1081-1091.

Tao G (2014) Multivariable adaptive control: A survey. Automatica 50(11): 2737 - 2764.

Williams EA, Shaw G and Elahinia M (2010) Control of an automotive shape memory alloy mirror actuator. Mechatronics 20(5): 527-534.

Young KD, Utkin VI and Ozguner U (1999) A control engineer's guide to sliding mode control. IEEE Transactions on Control Systems Technology 7(3): 328342.

Zheng T, Yang Y, Branson DT, Kang R, Guglielmino E, Cianchetti M, Caldwell DG and Yang G (2014) Control design of shape memory alloy based multiarm continuum robot inspired by octopus. In: 2014 IEEE 9th Conference on Industrial Electronics and Applications (ICIEA). pp. 1108-1113. 